\title{
Pore Structural Characterization of Fuel Cell Layers using Integrated Mercury Porosimetry and Computerized X-ray Tomography
}

Shoaib Malik ${ }^{\mathrm{a}, \#}$, Linda Smith ${ }^{\mathrm{b}}$, Jonathan Sharman ${ }^{\mathrm{b}}$, Elizabeth M. Holt ${ }^{\mathrm{c}}$, Sean P. Rigby $^{\mathrm{a}, *}$

aDepartment of Chemical and Environmental Engineering, University of Nottingham, University Park, Nottingham NG7 2RD, United Kingdom

bJohnson Matthey Technology Centre, Blount's Court Road, Sonning Common, Reading, United Kingdom.

${ }^{\mathrm{c} J o h n s o n}$ Matthey Process Technology Division, P.O. Box 1, Belasis Avenue, Billingham, Cleveland, United Kingdom

*Corresponding author

Current address: Department of Chemical and Environmental Engineering, University of Nottingham, U.K.

Telephone No.: +44 (0) 1159514078

Email: enzspr@exmail.nottingham.ac.uk

\#Present address: College of Sustainability Sciences and Humanities, Zayed University, Dubai, U.A.E. 


\begin{abstract}
The pore structure of the cathode catalyst layer of proton-exchange membrane (PEM) fuel cells is a major factor influencing cell performance. The nanostructure of the catalyst layer has been probed, using a novel combination of mercury porosimetry with computerised X-ray tomography (CXT), even though the nanopores were below the nominal CXT resolution. The method allows probing of the macroscopic spatial variability in the accessibility of the nanostructure. In particular, mercury entrapment within the catalyst layer showed a pronounced regular spatial patterning corresponding to the already higher X-ray absorbing regions of the fresh catalyst layer. The initial, greater X-ray absorption was due to a higher local concentration of carbon-supported platinum catalyst. This was due to segregation of ionomer away from these areas, caused by the particular screen printing catalyst layer deposition method used, which both enhanced the accessibility of the origin regions and, concomitantly, reduced the accessibility of the destination regions.
\end{abstract}

KEYWORDS: ink; mercury porosimetry; fuel cell; catalyst layer; pore accessibility; imaging 


\section{INTRODUCTION}

For proton-exchange membrane (PEM) fuel cells (FCs) the highest losses in cell voltage occur for the cathode-side catalyst layer. Hence, the performance of the cathode-side catalyst layer strongly affects the overall performance of the PEMFC. On the cathode side the reaction catalysed is the production of water from hydrogen nuclei migrating across the PEM and oxygen diffusing in from the exterior. The microstructure of the catalyst layer controls the rate of oxygen ingress and water removal, and, thence, the performance of the cathode layer. It is thus important to understand the structure of the cathode catalyst layer as it influences transport rates ${ }^{1}$.

The deposition method can significantly impact the microstructure of the cathode catalyst layer. Common deposition methods include spray-coating, gravure coating, slot die coating, screen printing, and ink jet printing of the ink containing the catalyst directly or indirectly onto the PEM polymer membrane. Previous work has shown that the pore structure of the catalyst layer affects its performance ${ }^{1}$. It is thus important to understand exactly what type of pore structure a particular fabrication process leads to. This work will consider a case study of a specifically engineered catalyst layer structure produced by screen-printing, a commonly employed laboratory-scale deposition technique. The catalyst layer produced during screenprinting is affected by the process parameters such as pressure, mesh size, scan speed and number of applications (screenings) ${ }^{2}$.

Computerised X-ray tomography (CXT) has previously ${ }^{3,4}$ been used to look at the agglomerate structure of the primary particles making up the catalyst layer, and at the secondary pore structure. The $50 \mathrm{~nm}$ resolution in this earlier work was not fine enough to distinguish 30-40 $\mathrm{nm}$ carbon support materials, 3-5 nm platinum nanoparticles, and 5-10 nm ionomer films within the agglomerates. However, CXT can be combined with other techniques to probe certain aspects of the smaller-scale nano-structure below the nominal CXT resolution limit. For example, CXT can be combined with mercury porosimetry, which can probe length-scales down to $\sim 3 \mathrm{~nm}^{5}$. Mercury porosimetry is based on the non-wetting nature of mercury to most materials, which means an increased hydrostatic pressure is 
required to force it into ever smaller pores. Once within small pores mercury can become entrapped even after the pressure is reduced back down to ambient. The high electron density of mercury makes it highly visible in CXT images. Hence, when entrapment occurs, CXT can be used to image the CXT resolution-scale spatial distribution of pores that mercury has entered at a given pressure. The integrated mercury porosimetry and CXT technique allows the study of variations in nanoscopic structure over macroscopic dimensions. This has the advantage, over other techniques that study nanoscale structure like electron tomography and dual-beam microscopy, that the sample volume is much larger and more likely to be statistically representative than the small volumes necessary for these other techniques. CXT, in combination with mercury porosimetry or other metal intrusion methods, has been used to study porous media previously. Mann and co-workers studied low melting point alloy intrusion into the macropore network of an alumina catalyst pellet using X-ray tomography ${ }^{6}$. Scheiba and co-workers used a Wood's metal intrusion process to study the electrode-gas diffusion layer (GDL) interface, though this method requires slicing and polishing sections and thus is destructive ${ }^{7}$. Hellmuth and co-workers studied mercury entrapped in macroporosity in rocks ${ }^{8}$. However, previous use of CXT combined with mercury porosimetry has generally looked at macroporosity, rather than lower scale properties of materials.

Mercury extrusion can be used to obtain further substantial information on the nature of a porous structure. It has been used to obtain pore connectivity ${ }^{9}$ and the macroscopic spatial arrangement of pore sizes ${ }^{10,11}$. This is because the entrapment process, which is a characteristic feature of extrusion in many materials, is particularly sensitive to specific aspects of the pore structure, such as the spatial relations of pores of different sizes and the inter-connectedness of the pore network ${ }^{12}$. In this work the entrapment process will be used to derive novel information about the spatial variation in pore accessibility. This paper describes a case study of the application of the integrated mercury porosimetry and CXT method to a screen-printed catalyst layer to demonstrate the capabilities of the technique in revealing issues with the pore structure. Complementary information was also obtained from electron microscopy techniques including imaging and Energy-dispersive X-ray spectroscopy (EDX). 


\section{METHOD}

\section{Material}

The sample was a prototype fuel cell layer with ink consisting of carbon-supported platinum and ionomer screen-printed onto a PTFE decal transfer sheet. The catalyst ink used to form the catalyst layers consisted of a $60 \% \mathrm{Pt}$ on carbon catalyst with an intentionally relatively poor Pt dispersion (to enhance $\mathrm{X}$-ray contrast) and aqueous Nafion solution to give a loading of $120 \%$ Nafion:C. The ink was prepared using a high shear mixing method to produce an ink with a smooth consistency, which was then coated onto a PTFE substrate by screen printing through a 123 mesh screen with a rubber blade squeegee. The coated PTFE samples were dried at $>110^{\circ} \mathrm{C}$, under conditions designed to deliberately form a specific catalyst layer structure with a preponderance of micro-cracking, to enable the capability of the integrated techniques to be best demonstrated.

\section{Electron Microscopy}

The machine used was a FEI XL30 FEG-ESEM. The SEM was done once for two different forms of the FC layer sample. The full FC layer sample with the PTFE backing layer was used for the first run. For the second run, the FC layer, i.e. carbon, platinum and ionomer binder, was scraped off from the backing layer (PTFE) to see structure at the back, and determine whether it was the same as the top surface. Both samples were placed on a stud and glued, and then placed in the SEM chamber. For the full sample a $10 \mathrm{keV}$ beam was used to minimise the distortion of the PTFE layer, which could be irradiated by higher voltages during scanning. For the scraped sample, since the PTFE was removed, the beam was increased to $15 \mathrm{keV}$ for increased resolution. Both experiments were done in a high vacuum environment.

\section{Energy-dispersive X-ray spectroscopy (EDX)}

The FEI XL30 FEG-ESEM was fitted with an Oxford Instruments ISIS unit to analyse the sample by EDX. The software SEMQuant was used to monitor the sample and the ZAF 
quantitative method was applied to smooth the sample signal. A system energy of $116 \mathrm{eV}$ was used. Randomly chosen spots were imaged at different magnifications and analysed. For each spot an energy-peak diagram was produced where each peak was measured against the energy indices within the software to identify the individual elements in the sample. Additionally, the software was used to quantify the element weight percentage and atomic percentage of each element in the sample.

\section{Mercury Porosimetry}

A Micromeritics Autopore IV 9500 machine was used which ran up to a pressure of 60,000 psia. The rig works in two stages where the first stage involves a low pressure stage and the second is a high pressure stage. The fuel cell layers were cut up into small slices and were weighed prior to inserting them as a 'swiss roll' form inside a penetrometer. The penetrometer was then sealed, lubricated and weighed again to gain a combined weight measurement of the whole penetrometer and the sample. The software was then run and input variables such as sample name, sample weight, temperature, mercury density and pressure point intervals were inserted. On finalizing these inputs the low pressure mercury intrusion stage was initiated. The software then recorded the volume uptake by the sample with increasing pressure. The low pressure stage involved evacuation to remove any air, moisture absorbed from the environment or any other liquids and unnecessary fluids which otherwise could create contamination issues or air pockets. The pressure of termination for the low pressure stages was $\sim 30$ psia. Once this first stage was completed the penetrometer was taken out and weighed again with the mercury inside. The compounded weight was then inserted into the software and the penetrometer was then placed into the high pressure port. The high pressure intrusion process was then initiated and the software continued recording the volume intruded into the sample up to 60,000 psia. The extrusion process was a reversal of the pressure direction to lower pressures. The experiment would terminate when the machine was unable to extrude any further mercury from the sample at the lowest pressure possible. Correction methods were applied by running a blank penetrometer. Thus any intrusion seen from the penetrometer, acting as a baseline, could then be removed from the sample data. The area of interest in the material was just the catalyst layer and so the experiment had to be performed twice on the two different materials in the layer. The first run was done using the backing layer only, and the second run was done on the area where the ink was printed onto the sheet. 
This was done because when the mercury intrudes into the catalyst layer, it also intrudes into the surface roughness of the backing and compresses the PTFE layer. Therefore, to remove the effects of the PTFE, the backing layer was analysed separately and the data could then be subtracted from the composite sample. This then provided the data corresponding to the mercury intruded into the catalyst layer only. Two repeats were done on different parts of the catalyst layer and two repeats were done on the backing layer.

\section{Computerised X-ray Tomography (CXT)}

Once the high pressure analysis was completed for the mercury porosimetry the sample was then removed from the penetrometer and cut into ten square pieces. Two square pieces were cut from a fresh sample and added to the stack. The layers were $\sim 2 \mathrm{~cm} \times 2 \mathrm{~cm}$. The stack, thus holding 12 layers, was then clamped by a sample holder to ensure that the alignment of the individual pieces would not be disturbed during the imaging process. The machine used for the X-ray imaging was a high resolution Xradia versa XRM-500 machine which can be operated at $160 \mathrm{kV}$ electrical power and current of $62 \mu \mathrm{A}$. The machine comprises an X-ray detector of a high end 1014×1014 pixel resolution with a 64 bit dynamic range. The pixel resolution was $1.93 \mu \mathrm{m}$. The sample and sample holder were placed onto a rotating stage between the beam source and the detector with a distance of $40 \mathrm{~mm}$. The stage rotated while X-rays passed through the sample and 5-6 images were then taken and recorded at each angular position. The computer software was then used to acquire data from the recorded images to reconstruct the entire sample. Options can be set such that the sample can be reconstructed in a $2 \mathrm{D}$ image sequence or $3 \mathrm{D}$ visual construct. The software of the machine was used to arrange the data in a stack of $2 \mathrm{D}$ images displaying the projections at every angular position. A total of $1013 \mu$-CT slices were produced. An open-access imaging processing software package, known as ImageJ, was then used to analyse the image stack to determine surface profiles and create 3D video simulations of the stack. Adjustments were made to how the stack was presented. The machine recorded the images in an angular sequence. This angular sequence was loaded into ImageJ and a second reconstruction and stack was generated which showed how the sample was orientated directly through the stack, rather than around the stack. The final image sequence was thus shown to go through the stack layer-by-layer rather than at a significant angle. Each layer (backing and ink) had a 
thickness equivalent to typically a stack of 75 CXT image slices. Each catalyst layer typically was encompassed by 25 CXT image slices, on average.

\section{RESULTS}

Figure 1 shows a general overview of the uppermost surface of the catalyst layer. It can be seen that the surface has many crescent-shaped cracks. Figure 1 also shows close-up views of a crack region and the interior of a crack. The close-up views revealed some white spots which were studied in more detail. 
Figure 1: Electron micrographs of fuel cell layer sample, showing (a) general overview of ink upper surface, (b) close-up view of ink surface and crack, and (c) view of interior of a crack.

(a)

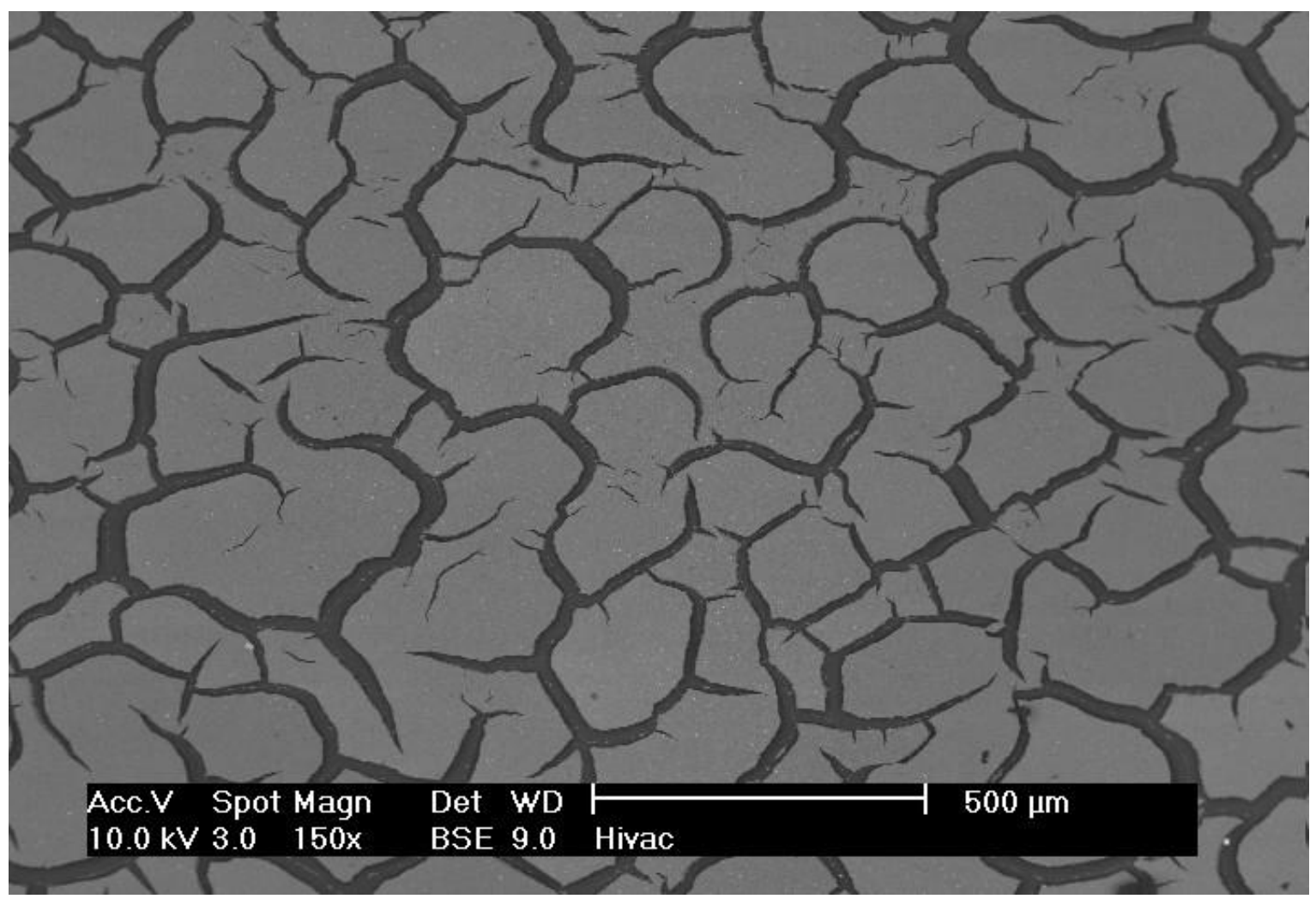

(b)

(c)

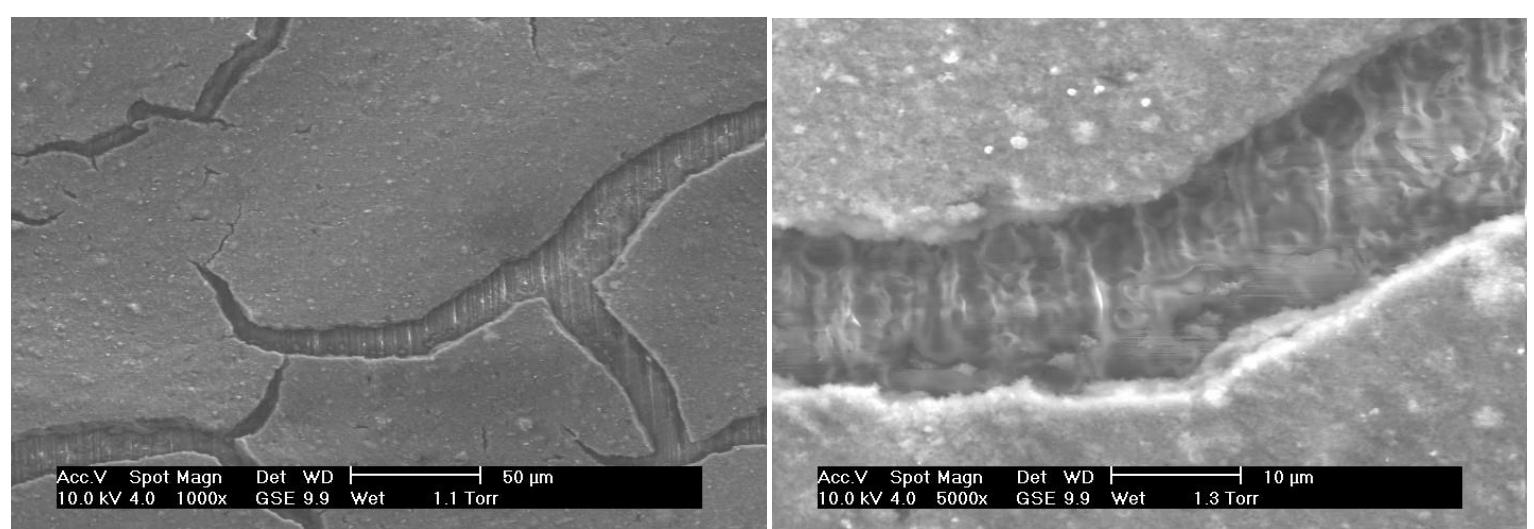


Figure 2 shows close-up views of the white spot regions revealed on the wider SEM views in Figure 1. Both back-scattering and secondary electron imaging were used. Secondary electron imaging shows the morphology of the surface, whereas back-scattering electron imaging displays contrast by composition based on the constituent elements in the sample. The brightness of the white regions in the back-scattering electron images suggests the presence of a high atomic number element, which must be platinum. The rough morphology of the darker background underlying the white clusters in the close-up views is suggestive of porosity on the nanometre length-scale in the substrate. 
Figure 2: Views of clusters of platinum on ink surface. Images include a comparison of a backscattered electron image (a) and secondary electron image (b) of the same cluster, together with close-ups of the cluster with ever increasing magnification (c and d). The images also show a comparison of a backscattered electron image (e) and secondary electron image (f) of another cluster.

(a)

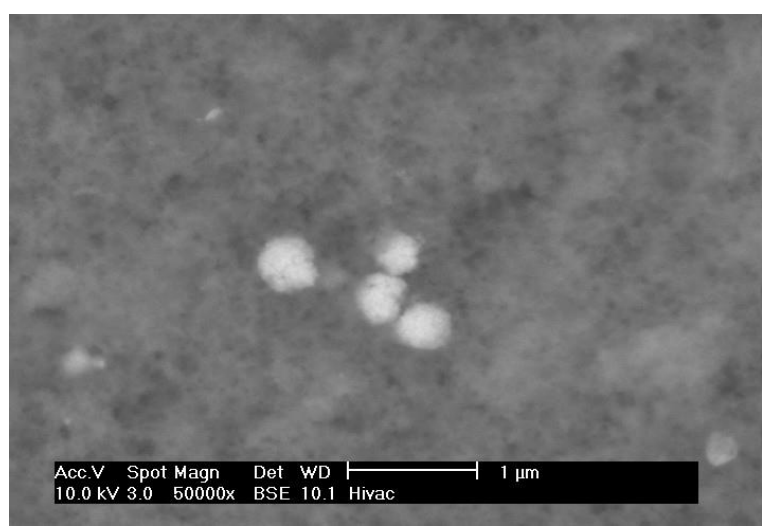

(b)

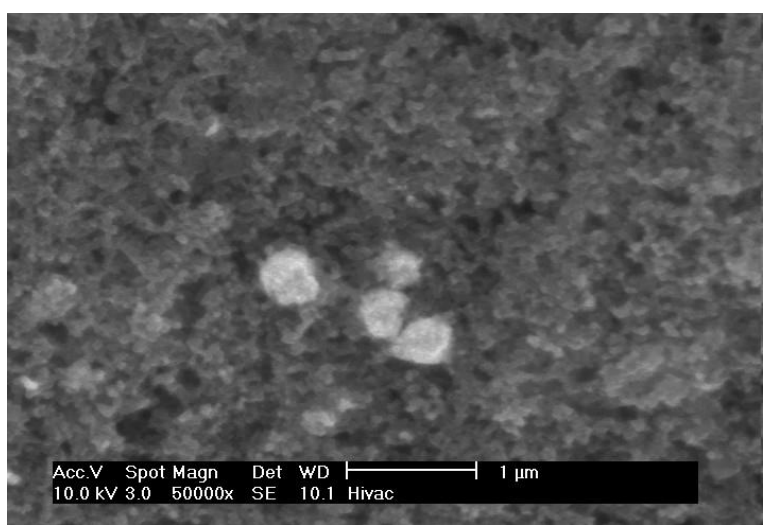

(c)

(d)
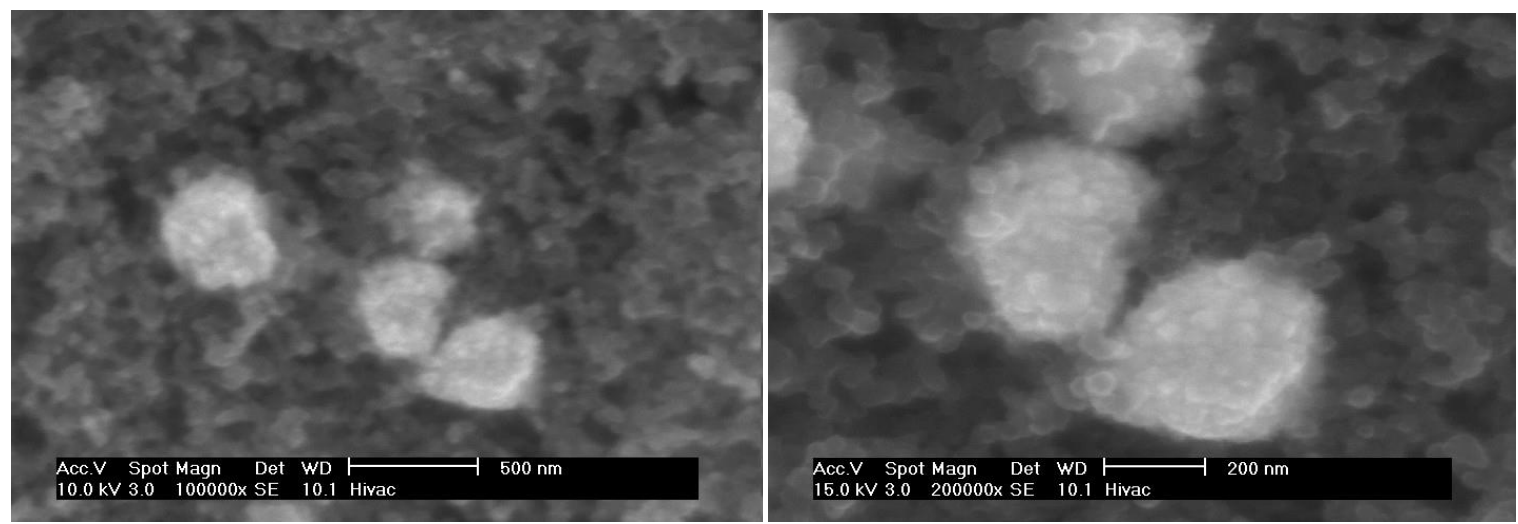

(e)

(f)
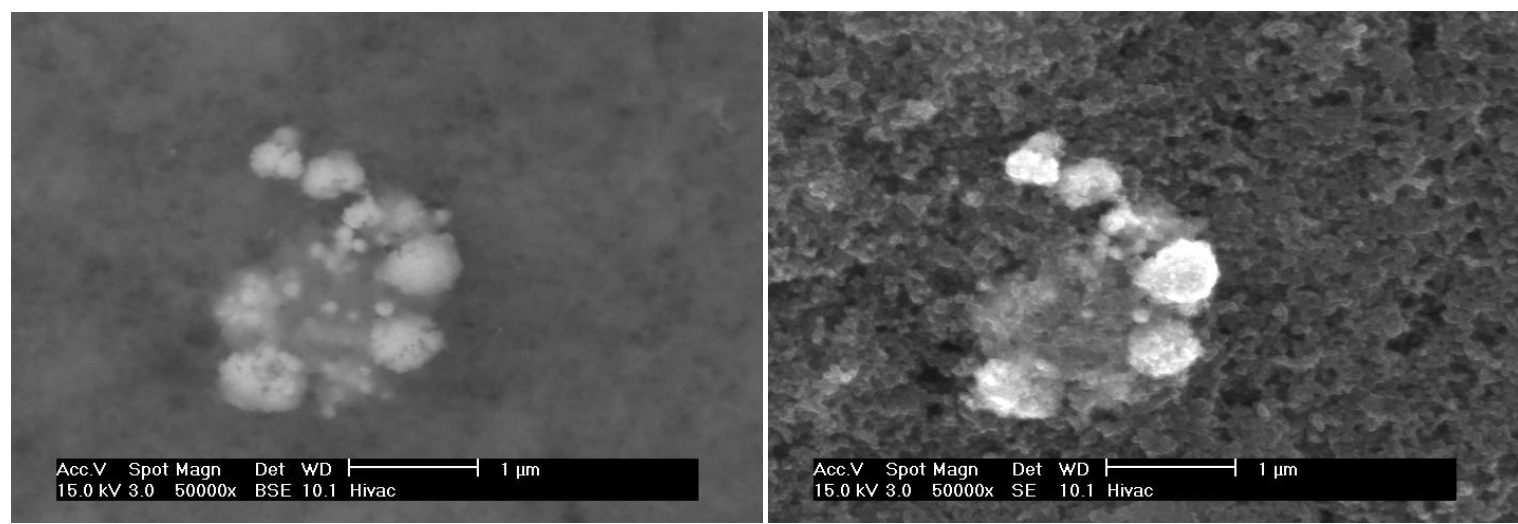
Figure 3 shows relatively low resolution overview images of the cross-section through the thickness of the catalyst layer. Figure 3 also shows close-up views using both secondary electron and back-scattered electron imaging. The low resolution images show that there is some variation in the overall thickness of the catalyst layer but it is not regular and periodic. The close-up views do not reveal any obvious regular variation in solid density. However, the secondary electron image does reveal the presence of slit-shaped pores orientated parallel to the faces of the catalyst layer. This might be suggestive of some underlying lamellar structure in the fuel cell layer. The back-scattered electron image shows bright white spots distributed through the layer suggesting that platinum clusters occur throughout the thickness of the ink layer. 
Figure 3: Back-scattered electron SEM images of typical cross-sections through the ink layer (a-d), together with a secondary electron close-up view of part of a cross-section (e) and a back-scattered electron image of the same close-up view (f).

(a)

(b)
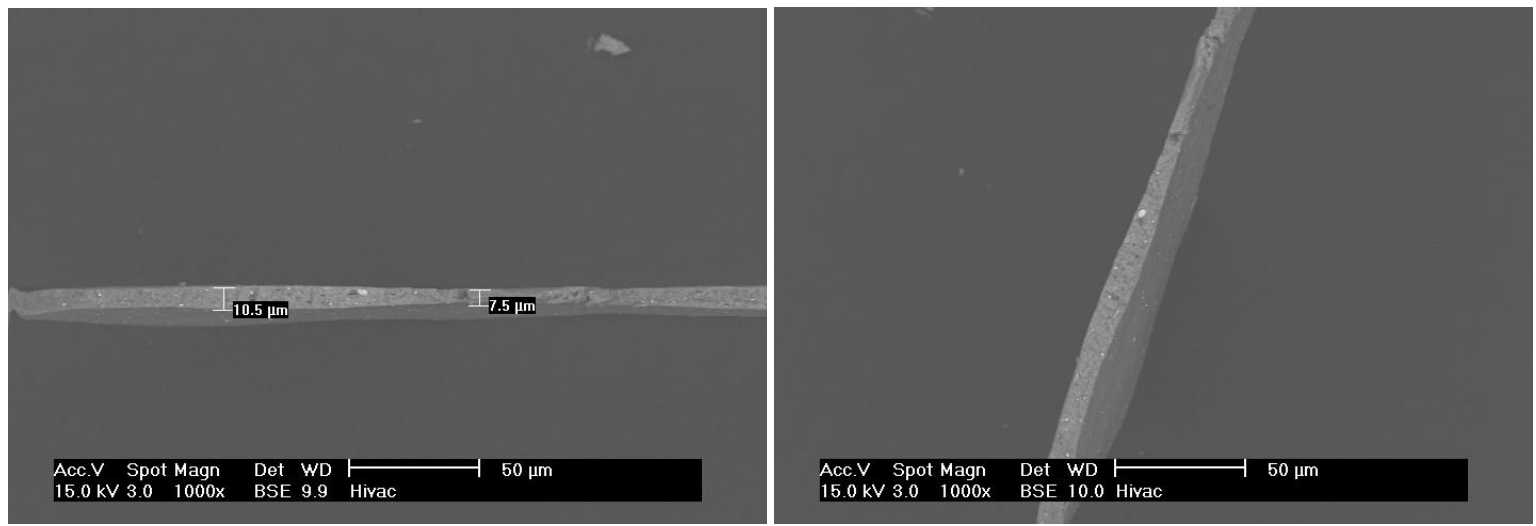

(c)

(d)
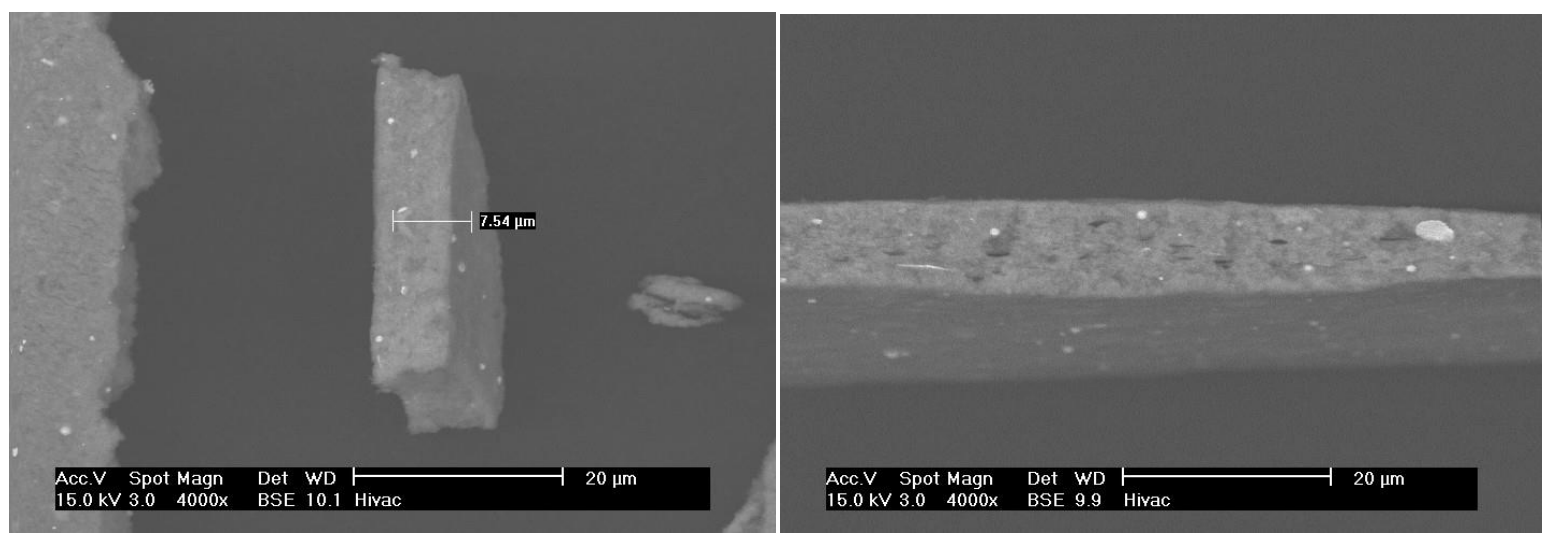

(e)

(f)
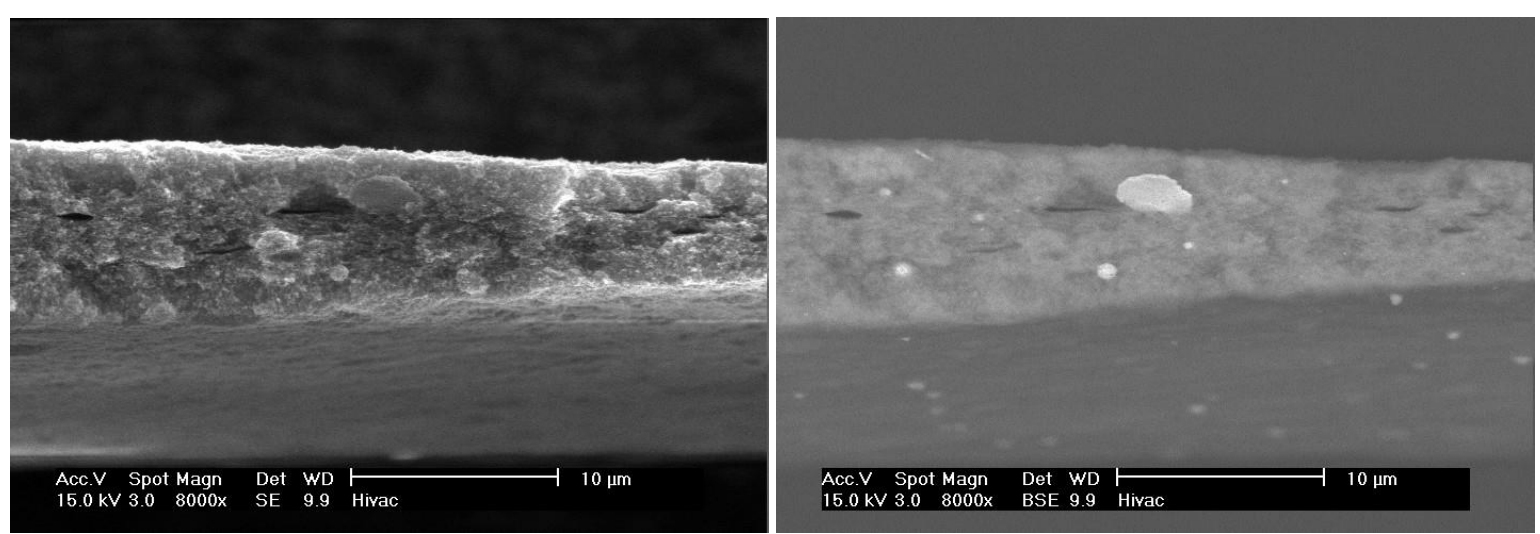
Figure 4 shows the data for a typical region of the ink layer examined using EDX. Table 1 shows the elemental and atomic abundances by percentage for carbon, fluorine, and platinum in the regions shown in Figure 4. The set of images in Figure 4 consists of a series of imageacquisitions with increasing magnification, focussing down upon a particular platinum cluster. From the spectra in Figure 4 and the abundances in Table 1 it can be seen that as the image is focussed ever closer onto the platinum cluster the fluorine signal declines relative to the platinum. This suggests less fluorine in the regions occupied by platinum clusters.

Table 1: Elemental and atomic abundances of carbon, fluorine and platinum for the regions of interest corresponding to the series of images in Figure 4.

\begin{tabular}{|l|l|l|l|}
\hline Figure & Species & $\begin{array}{l}\text { Elemental } \\
\text { abundance/\% }\end{array}$ & $\begin{array}{l}\text { Atomic } \\
\text { abundance/\% }\end{array}$ \\
\hline $4 \mathrm{a}$ & Carbon & 30.87 & 63.49 \\
& Fluorine & 23.65 & 30.75 \\
& Platinum & 45.49 & 5.76 \\
\hline $4 \mathrm{c}$ & Carbon & 33.85 & 67.15 \\
& Fluorine & 21.87 & 27.44 \\
& Platinum & 44.28 & 5.41 \\
\hline $4 \mathrm{e}$ & Carbon & 33.92 & 78.94 \\
& Fluorine & 8.74 & 12.85 \\
& Platinum & 57.34 & 8.21 \\
\hline
\end{tabular}


Figure 4: SEM images ((a), (c) and (e)) and corresponding EDX spectra ((b), (d) and (f)) of ever higher magnification of region of ink containing platinum clusters. The scale bars in (a) and (c) corresponds to $20 \mu \mathrm{m}$, while that in (e) corresponds to $2 \mu \mathrm{m}$.

(a)

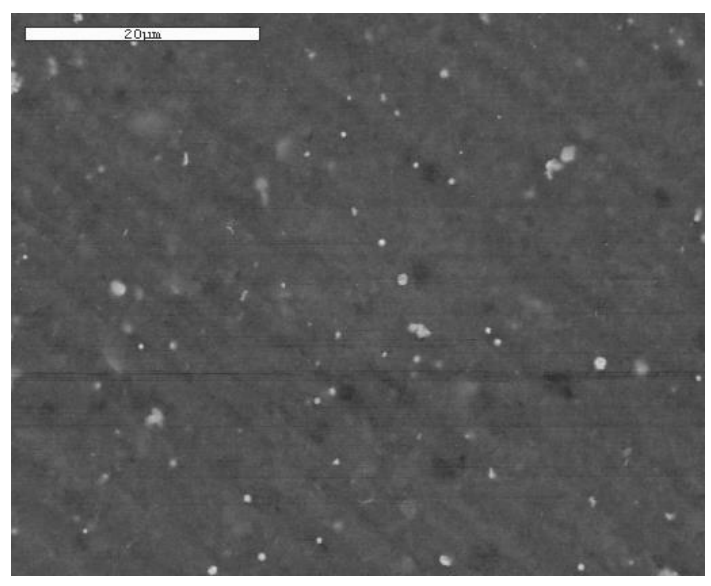

(c)

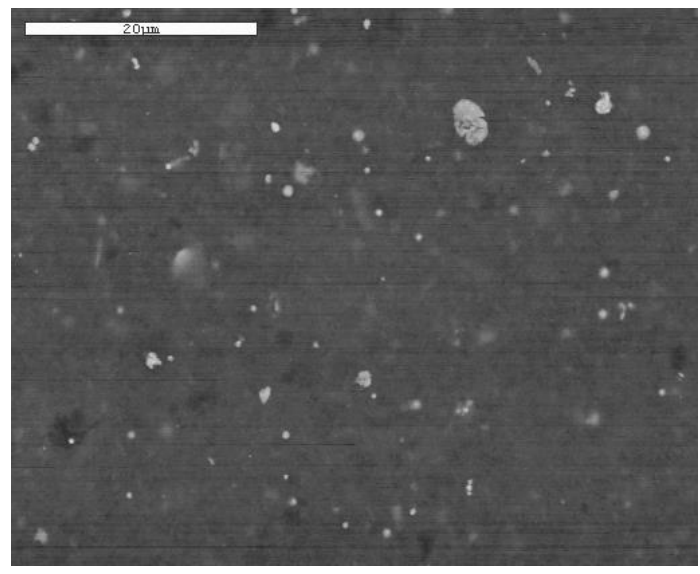

(e)

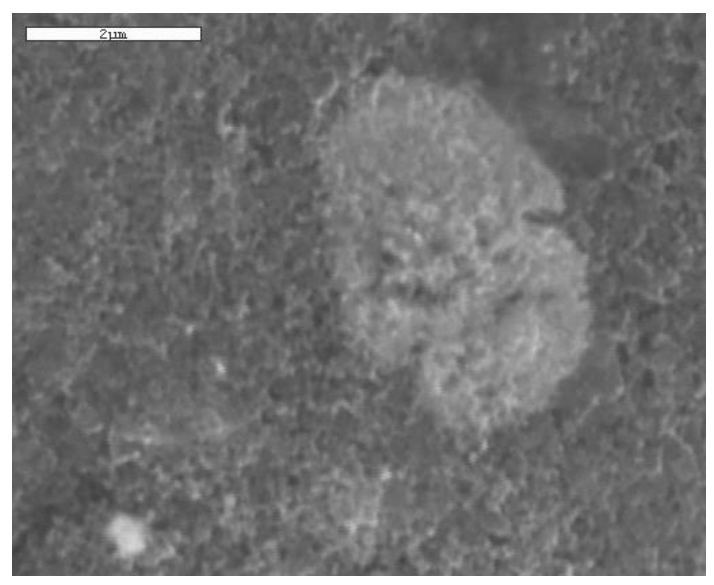

(b)

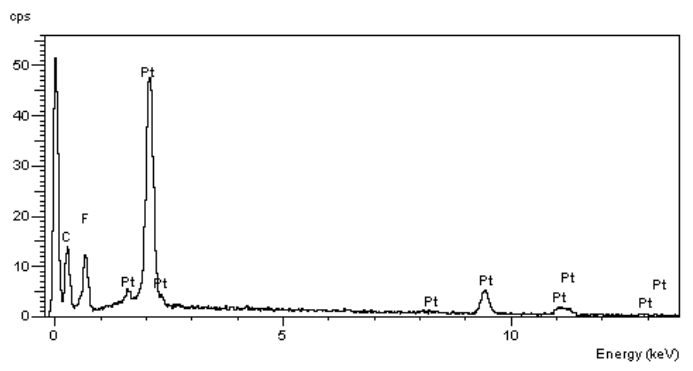

(d)

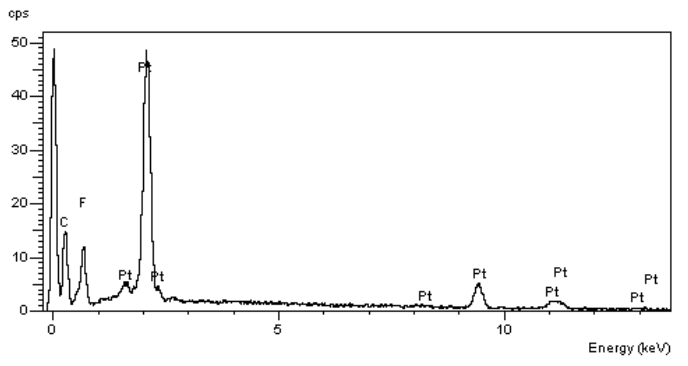

(f)

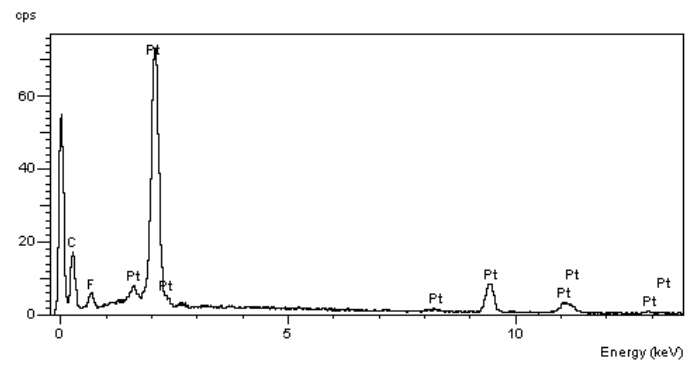


Figure 5(a) shows the resultant mercury intrusion and extrusion curves derived just for the mesopore region of the catalyst layer whilst still in-situ on the backing, by subtraction of the data for just the PTFE backing from the data for the composite (ink on backing) FC layer. The intrusion and extrusion pressures have then (Figure 5(b)) been converted to pore radius using the standard Kloubek ${ }^{13}$ correlation, which removes the effects of contact angle hysteresis. From Figure 5 it can be shown that the modal intrusion radius is $\sim 10-20 \mathrm{~nm}$. This would correspond to the typical neck size at the entrance to the ink layer mesopore network accessible from just the front of the complete composite FC layer. This is the side that would be bound to the fuel cell membrane during a typical lamination process. It can also be seen that, once the contact angle hysteresis is removed, and allowing for random error arising from subtraction of the backing data, intrusion into the ink layer itself is completely reversible for the smallest pore sizes, i.e. smaller than $\sim 6-7 \mathrm{~nm}$. The lack of mercury entrapment in the smallest pores has been confirmed by mercury thermoporometry of the entrapped mercury (data not shown). Hence, mercury entrapment is confined to pores of roughly modal size and larger. It can be seen that the overall entrapment level (residual mercury saturation) is high.

Given the intrusion at the highest pressures does not lead to entrapment, which would provide direct evidence of actual mercury penetration, it might be suggested that that intrusion is only apparent, and is really due to compression of the sample. However, any apparent intrusion cannot be simple, reversible, elastic compression of the pore walls because there is still some hysteresis at the highest pressures. In addition, dimensional change has been suggested previously as a cause of hysteresis in porosimetry when mercury pressure is exerted on the exterior wall of a still to-be-intruded pore ${ }^{14}$. The strain of a given pore, that could give rise to the particular width of the hysteresis, is inversely proportional to the modulus of elasticity of the walls ${ }^{14}$. However, given that the Kloubek correlations were obtained using silica (CPG) and the material under test here is carbon, it seems unlikely that the Kloubek correlations ${ }^{13}$ would be able to achieve superposition of the intrusion and extrusion curves if the hysteresis had a significant contribution due to compression, because of the difference in moduli of elasticity of the two materials. Further, the anticipated absolute value of the strain for the pores of a silica material, with modulus of elasticity of $\sim 10^{11} \mathrm{~Pa}$, at the highest possible pressure $(414 \mathrm{MPa})$ in the mercury porosimeter used is $\sim 10^{-3}$, which is not big enough to manifest in the porosimetry experiment. Graphitized carbon blacks have values of modulus of elasticity of similar order of magnitude to silica, and thus similar tiny sizes of strain. In 
addition, experiments with carbons that have larger amounts of mesopore volume in the range 2-6 nm show more apparent intrusion of mercury than those carbons with lower mesopore volumes in this range, where the mesopore volumes were determined by nitrogen sorption and analysis with Micromeritics (Saieus) software. If the volume change was an artefact due to compression, this might be expected to be similar for all the carbon blacks.

Figure 5: (a) Mercury porosimetry intrusion (solid line) and retraction $(\times)$ curves for a complete FC layer on PTFE backing, after subtraction of a blank run with just PTFE backing.

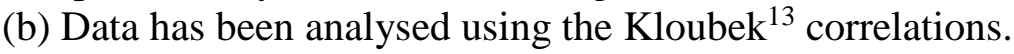

(a)

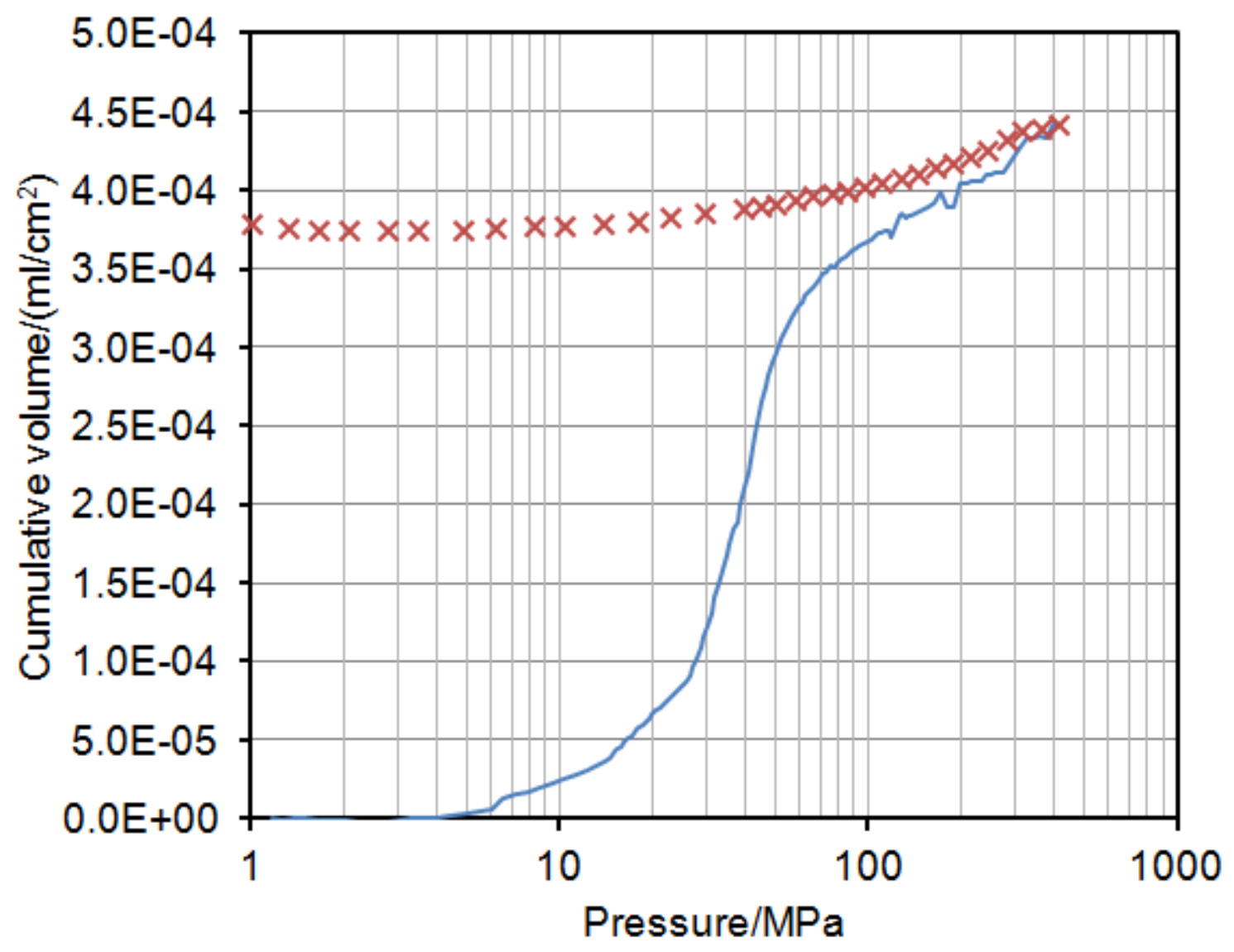


(b)

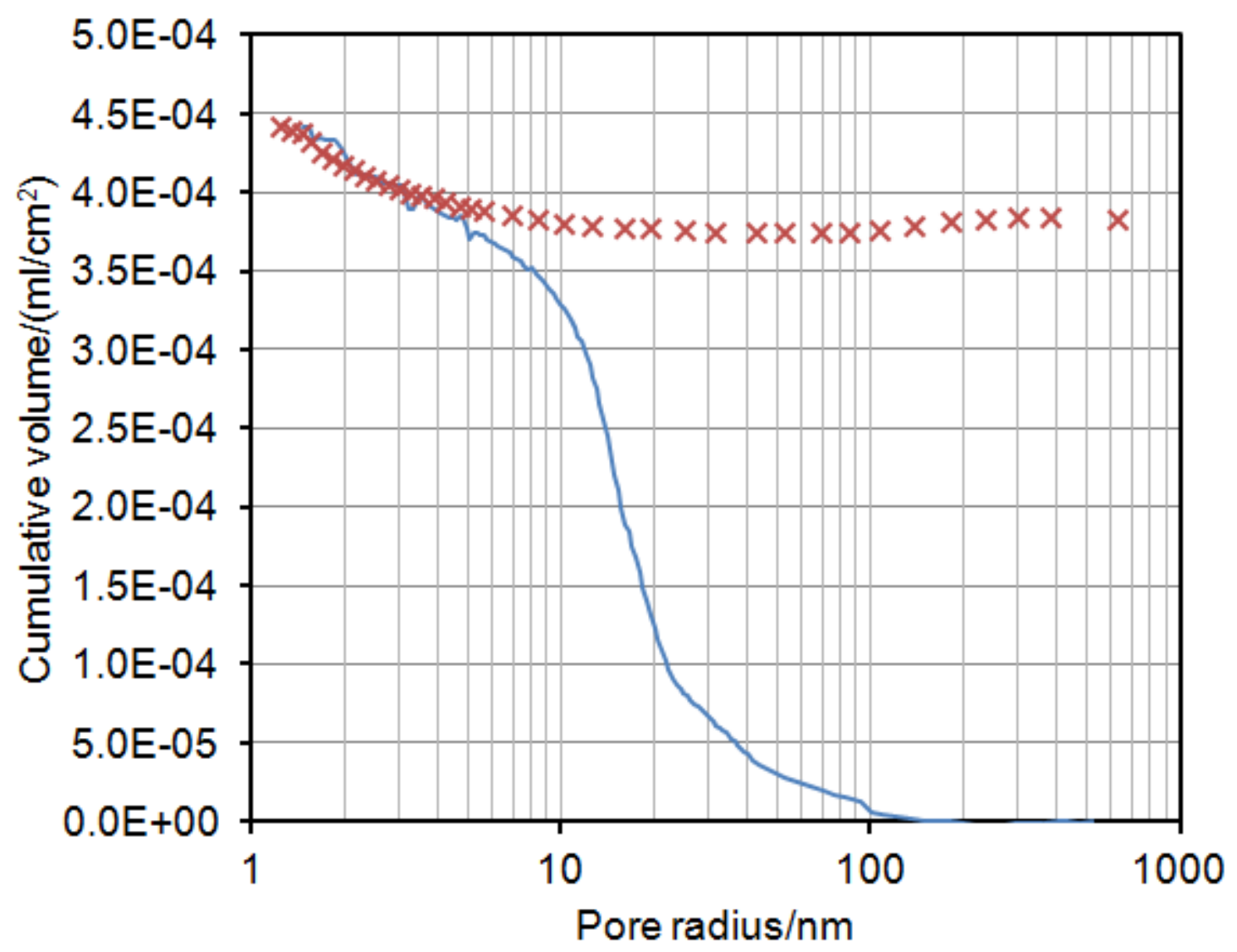

Figure 6 shows electron micrographs of general overviews of the top surface of the fuel cell layer following mercury porosimetry. It can be seen that mercury is retained in the crescentshaped macroscopic cracks that cover the surface even though they have direct access to the exterior. This is because atmospheric pressure is sufficient to keep mercury in pores of sizes 14 microns or larger. In some cases, perhaps due to inadvertent flexing of the layer, mercury has left the macropores. 
Figure 6: SEM images of general overview of upper surface of ink layer following mercury porosimetry experiment (a), and a region where some mercury had exited from some cracks (b).

(a)

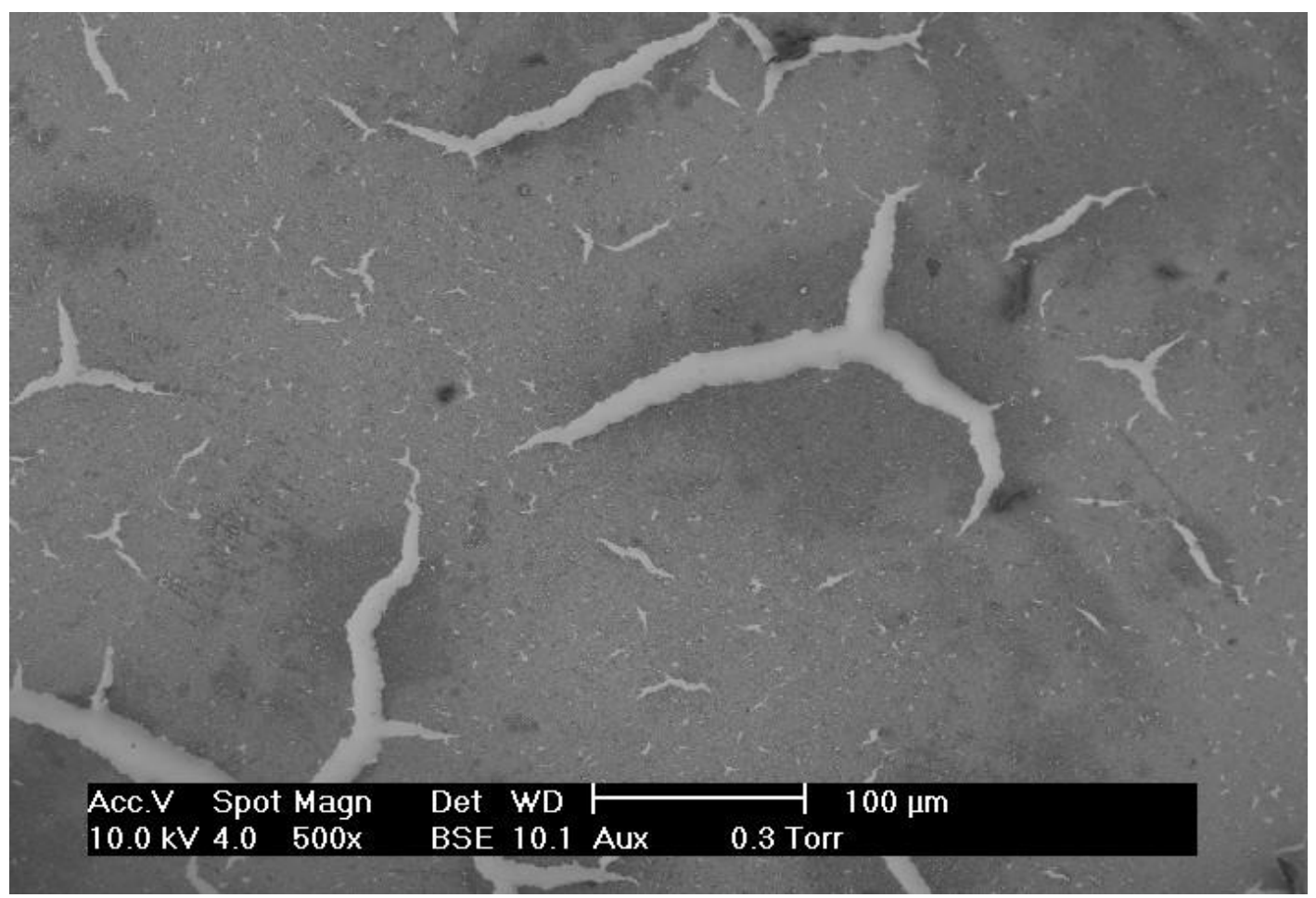

(b)

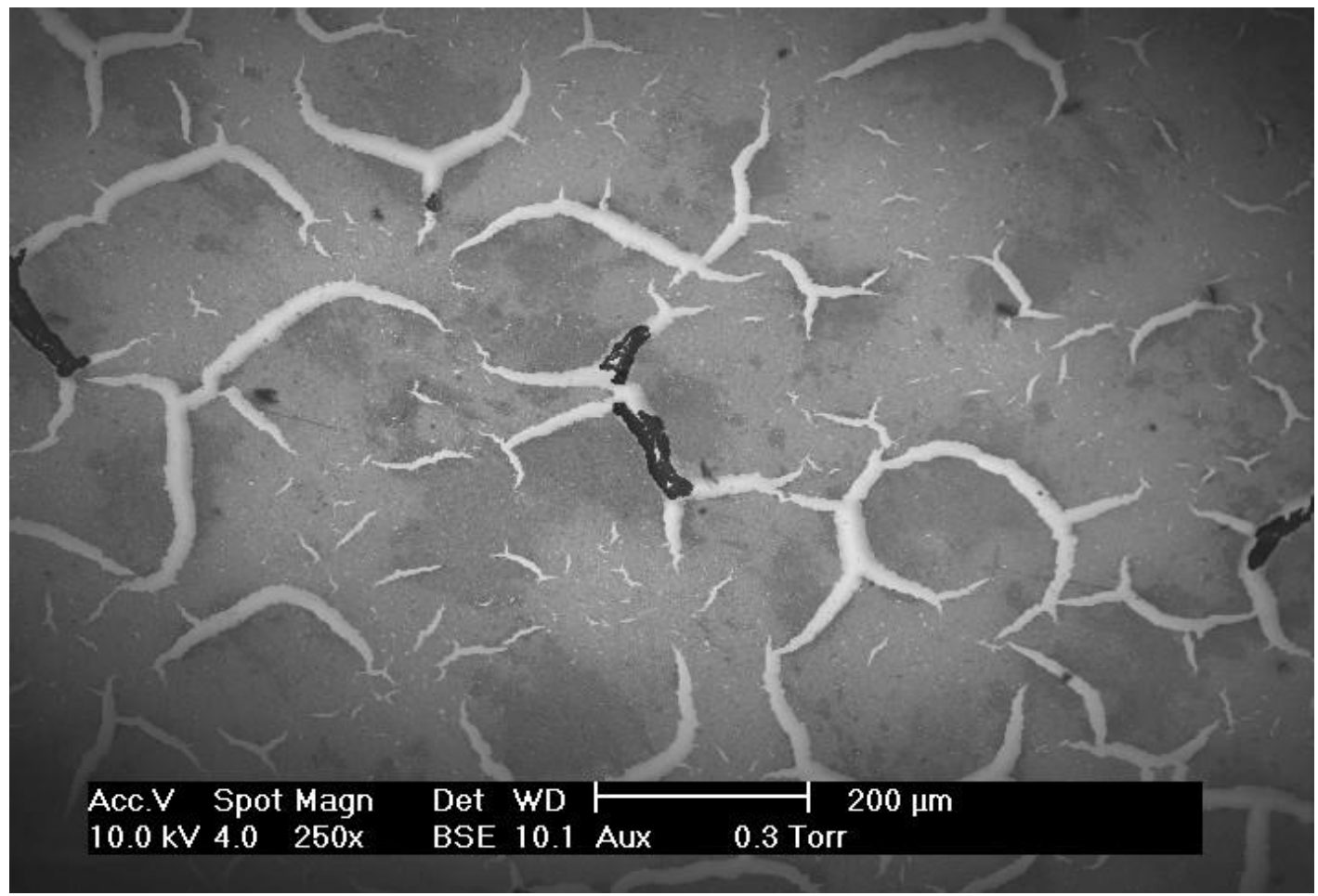


Figure 7 shows cropped parts of successive ((a) followed by (b)) CXT image slices for roughly the same spatial region of a particular fuel cell layer following porosimetry. In the CXT images the darker regions correspond to low density and the brighter, whiter regions to high density. The images show a periodic 'polka dot' like pattern of dark, low density regions separated by brighter, high density regions. The pattern of white spots is similar to the array of spheres in a single layer of hexagonal close packing. In the images in Figure 7, obtained following mercury entrapment, the mercury trapped in the macroscopic crescent-shaped cracks can be seen superimposed on the regular array of black and white spots. The images also show a crack that has partially lost its mercury from a middle segment, which now has very low intensity in the image. As the electron micrographs in Figure 1 have shown, the cracks tend to penetrate completely through the ink layer to the PTFE backing. It is noted that the slice thickness of $1.93 \mu \mathrm{m}$ is about a quarter of the size of the thinnest sections of ink layer seen in Figure 3. The images show (just below and left of centre) bright white crescentshaped cracks full of mercury crossing a black 'polka dot' low density region. This feature was evident in the corresponding spatial location for five successive image slices, including the two slices shown in Figure 7. The electron microscopy data has shown that the cracks traverse the full thickness of the ink layer, and the mercury fills these cracks. Hence, the presence of mercury in a crack in the image means the image slice is located within the ink layer. The aforementioned finding thus suggests that the low density spots in the image do not arise because the volume of the image slice encompasses part of the void above a depression in the surface of the ink layer, at a point where that layer had narrowed in thickness to less than the image slice.

Figure 8 shows typical examples of other image slices through a fresh fuel cell layer and through another layer containing entrapped mercury. The images are for slices where the orientation of the image plane closely matches the mid-plane of the catalyst layer. From Figure 8 it can be seen that the fresh fuel cell layer again has a periodic array of black spots and white spots akin to a 'polka-dot' like pattern. From Figure 8, it can be seen that, along the intensity profiles for the fresh sample, the intensity varies from $\sim 60-80$ in the black spots to 90-100 at the white spots. The intensity profile for the images containing entrapped mercury shows that the intensity of the black spot regions has stayed at $\sim 60-80$, while that of the white spots has increased to $\sim 100-120$. It is noted that the intensity of each peak and trough in the intensity profile is very similar and shows no systematic decrease from peak-to-peak as might be expected if the image slice was tilted and was missing part of the ink layer towards one 
side. The very high intensity spikes in the intensity profile correspond to the mercury entrapped in the macroscopic crescent-shaped cracks. The results suggest that the 'polka-dot' pattern in the CXT images has become more defined because the intensity of the high density spots has increased further following mercury entrapment. Since mercury is a dense element and is likely to increase absorption, and thus intensity, as it has for crescent-shaped cracks, then the increase in intensity in the white spot regions must be caused by higher mercury entrapment in those regions. 
Figure 7: Cropped successive ((a) followed by (b)) CXT image slices for corresponding similar spatial region of a particular fuel cell layer showing (just lower left of centre) bright white crescent-shaped cracks full of mercury crossing a black 'polka dot' low density region. The scale bar at the bottom left of each image corresponds to $500 \mu \mathrm{m}$.

(a)

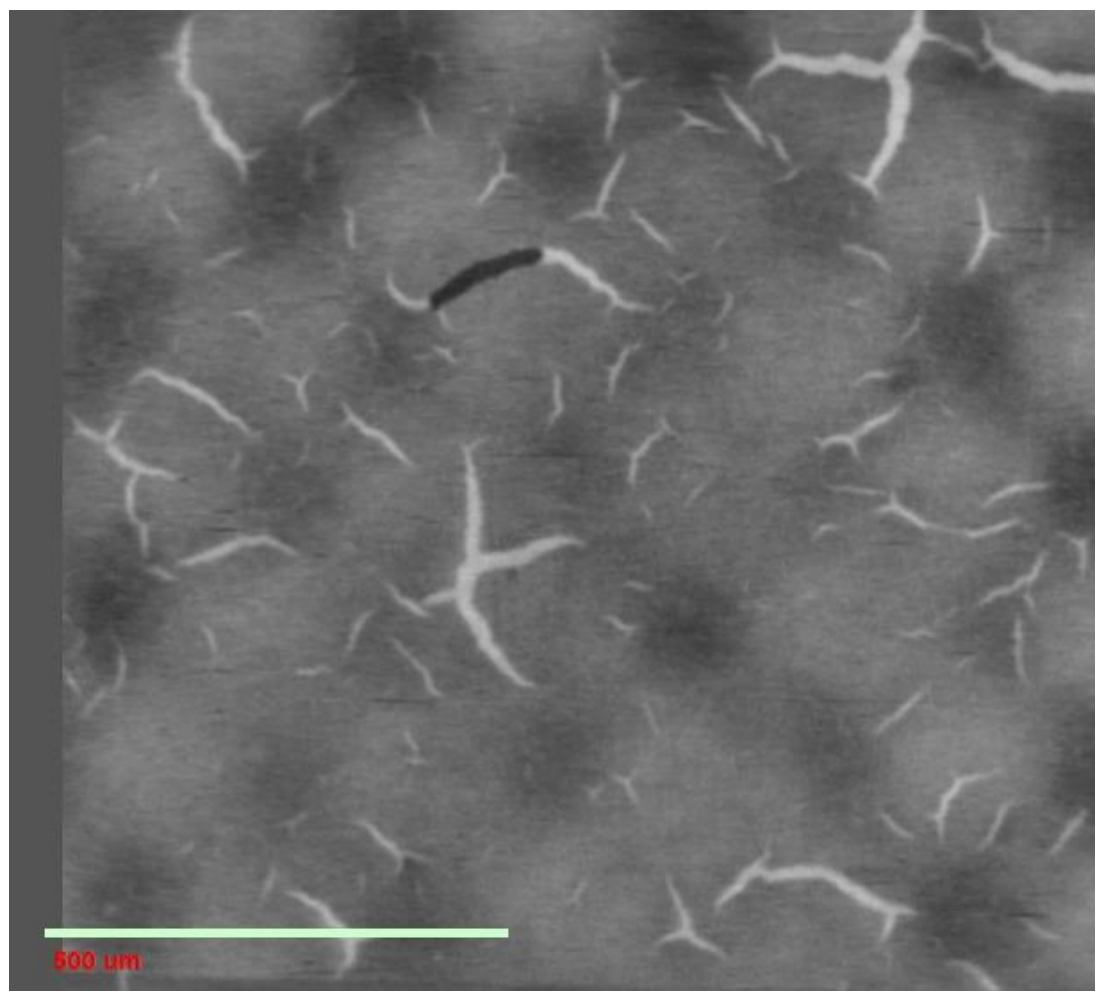

(b)

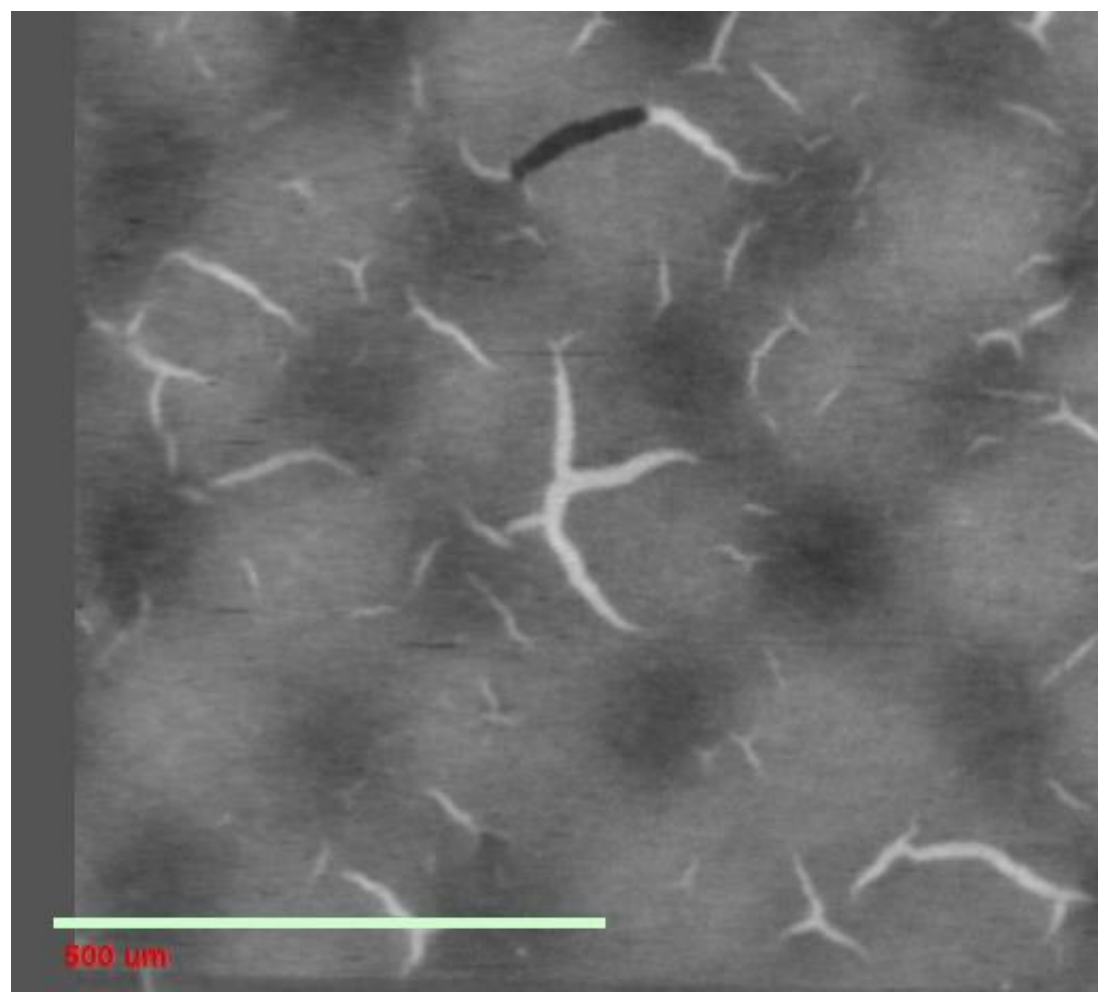


Figure 8: CXT reconstructed image slices of a section of fresh fuel cell layer (a and c) and a section of fuel cell layer following mercury porosimetry (e and $\mathrm{g}$ ). The brighter white regions correspond to high density regions with high $\mathrm{x}$-ray absorption and the dark regions correspond to low density regions with low $\mathrm{x}$-ray absorption. Each image slice has a horizontal line in the upper zone which corresponds to the track across which the linear intensity profiles were obtained before porosimetry ( $b$ and d) and after porosimetry ( $f$ and $h$ ). The scale bar at the bottom left of each image corresponds to $500 \mu \mathrm{m}$.

(a)

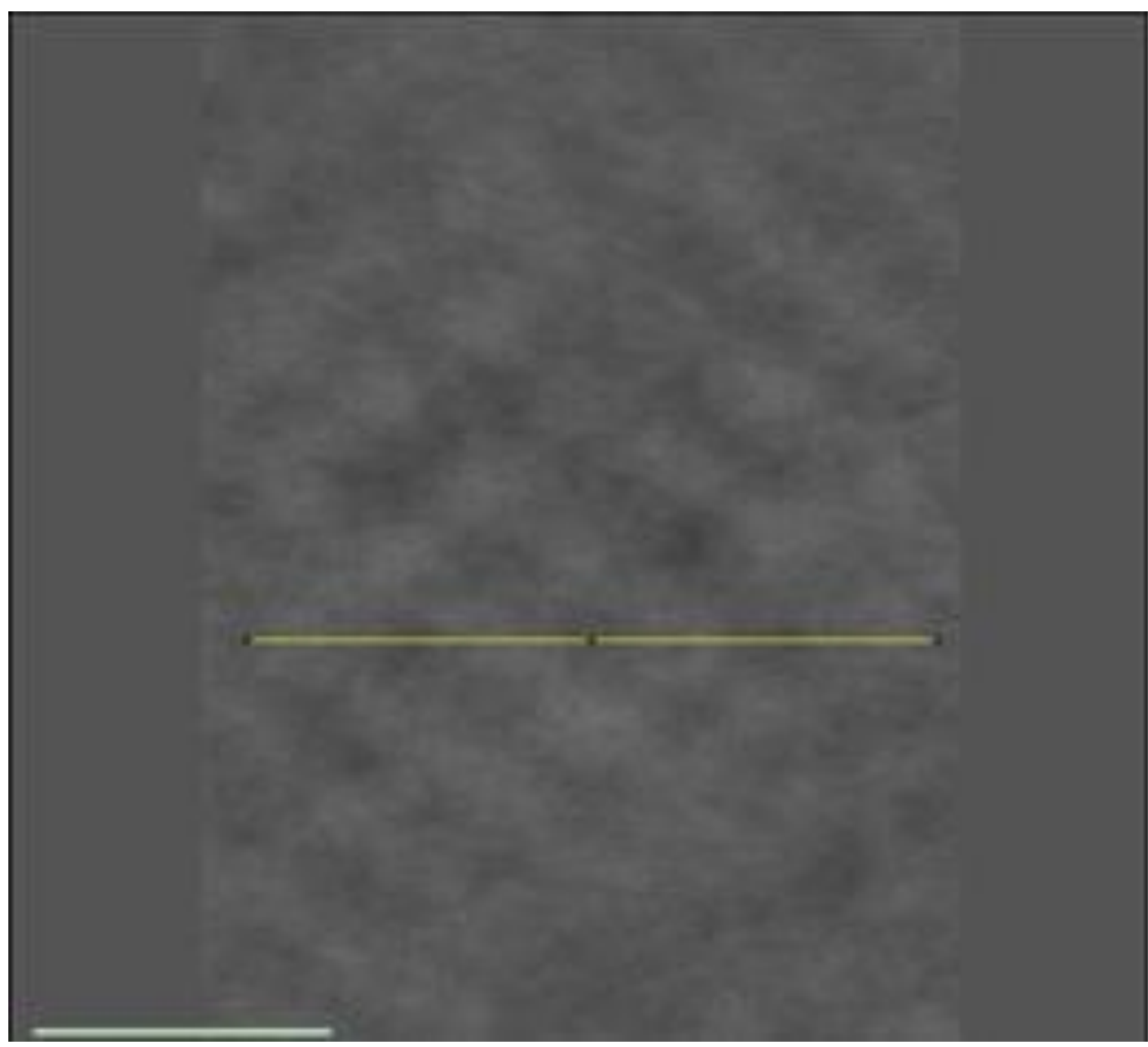

(b)

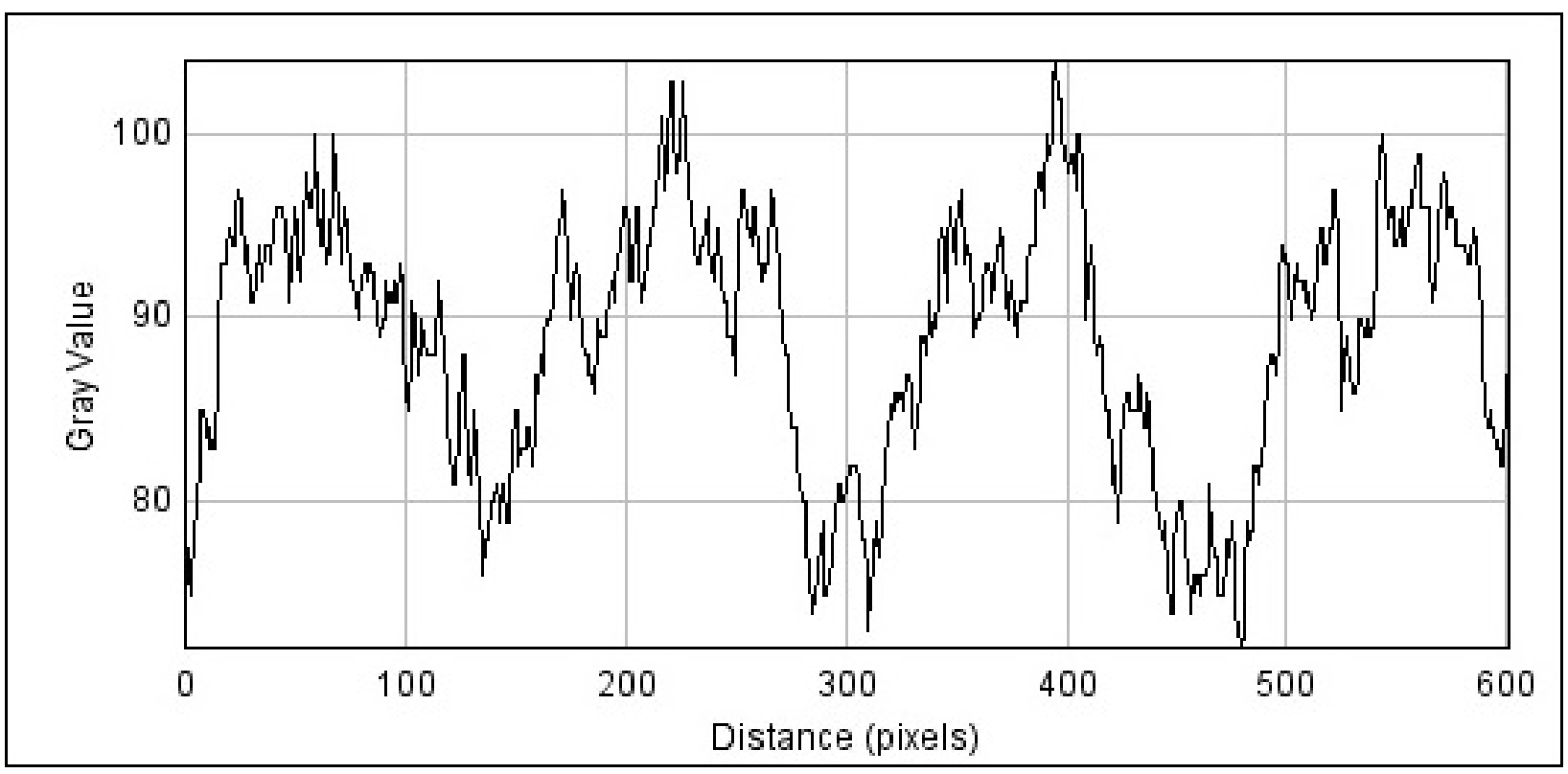


(c)

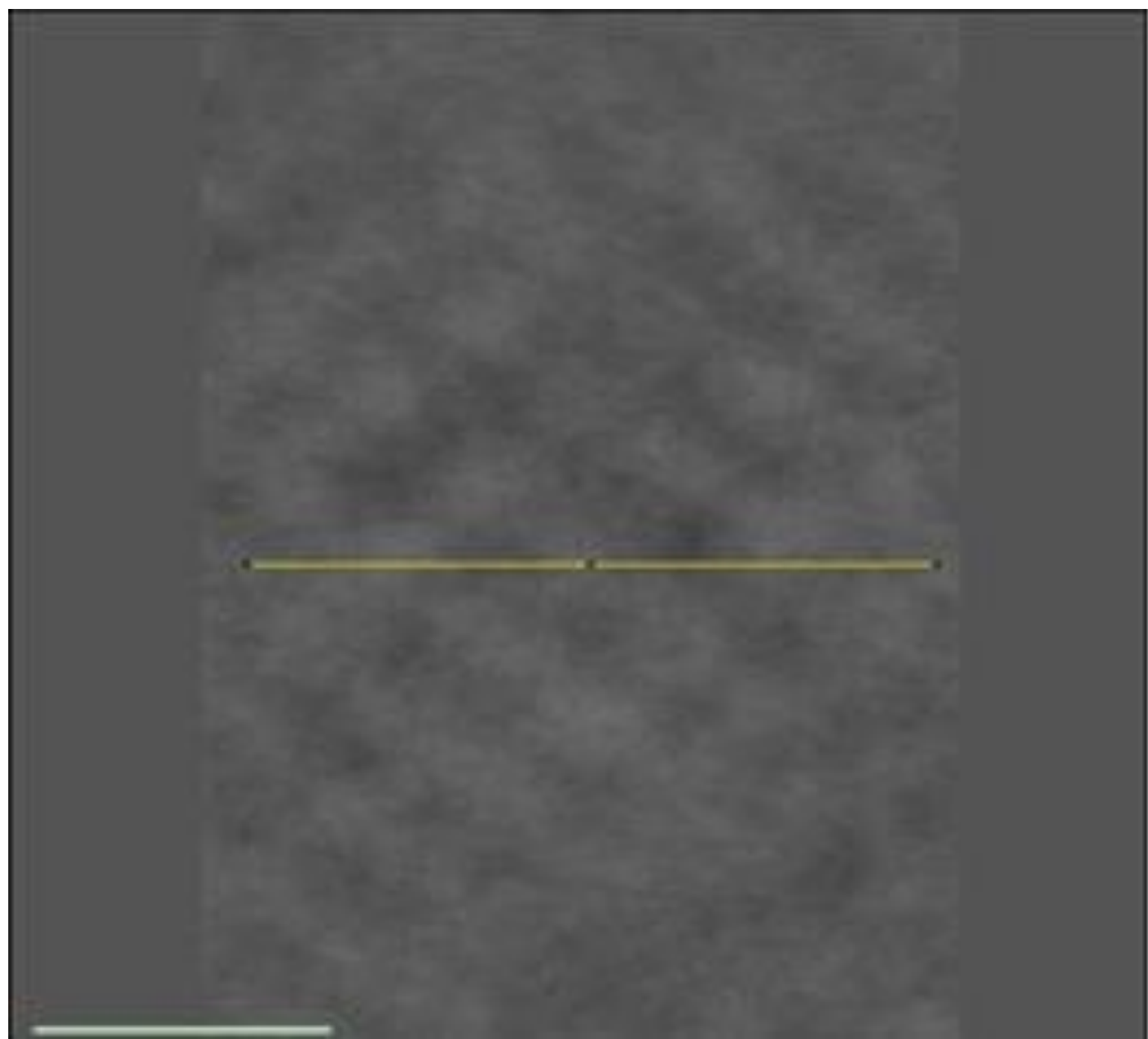

(d)

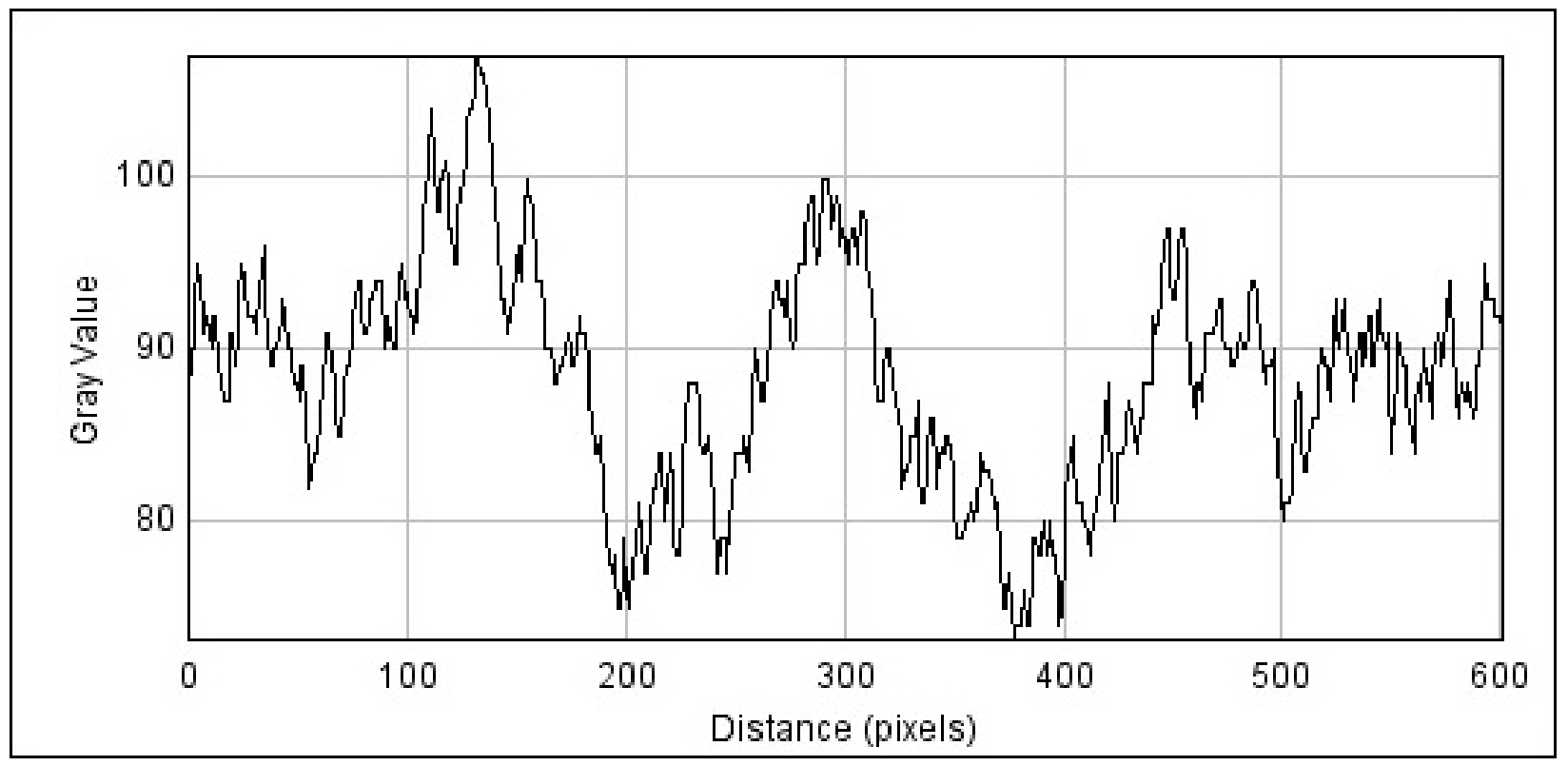


(e)

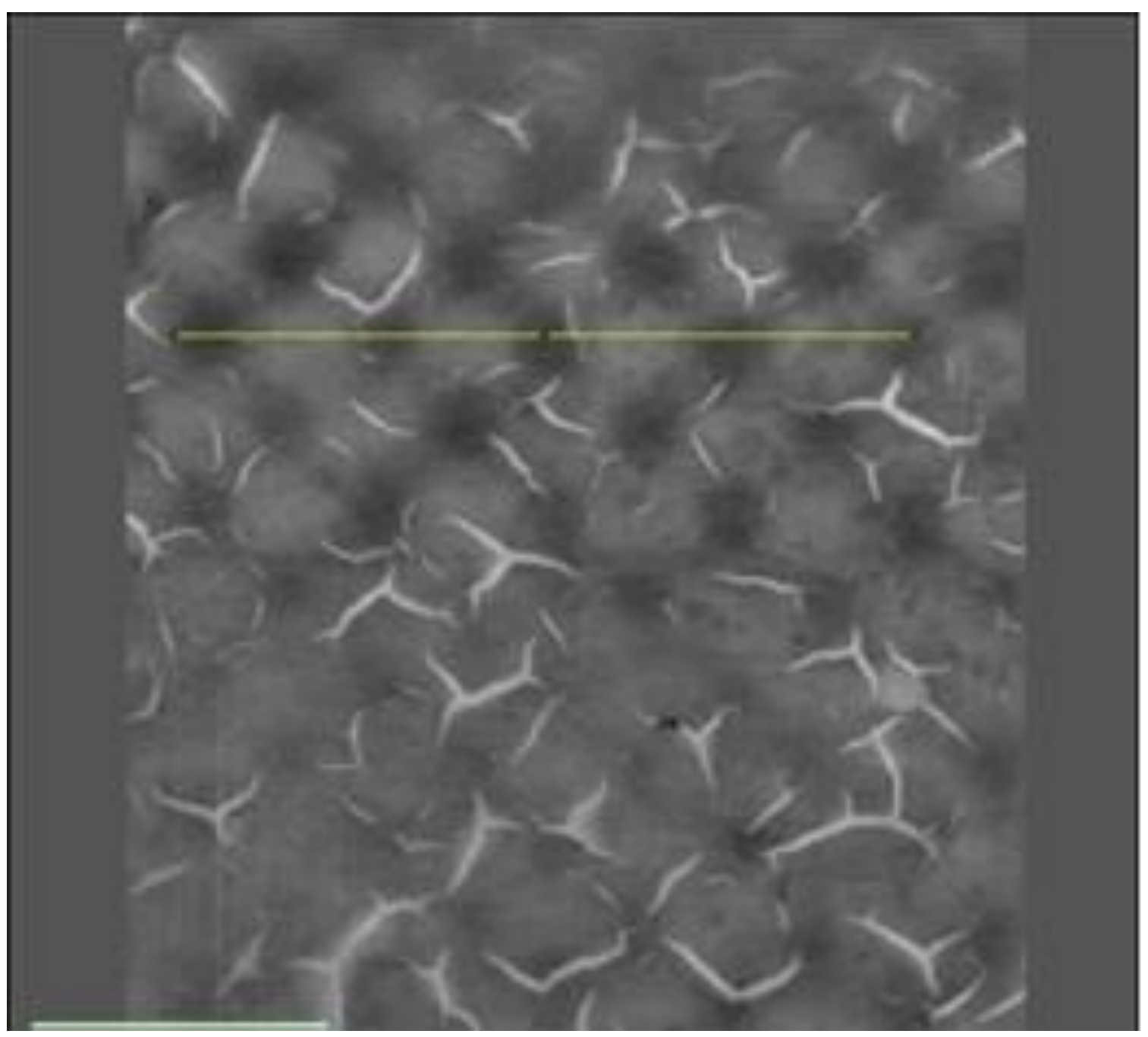

(f)

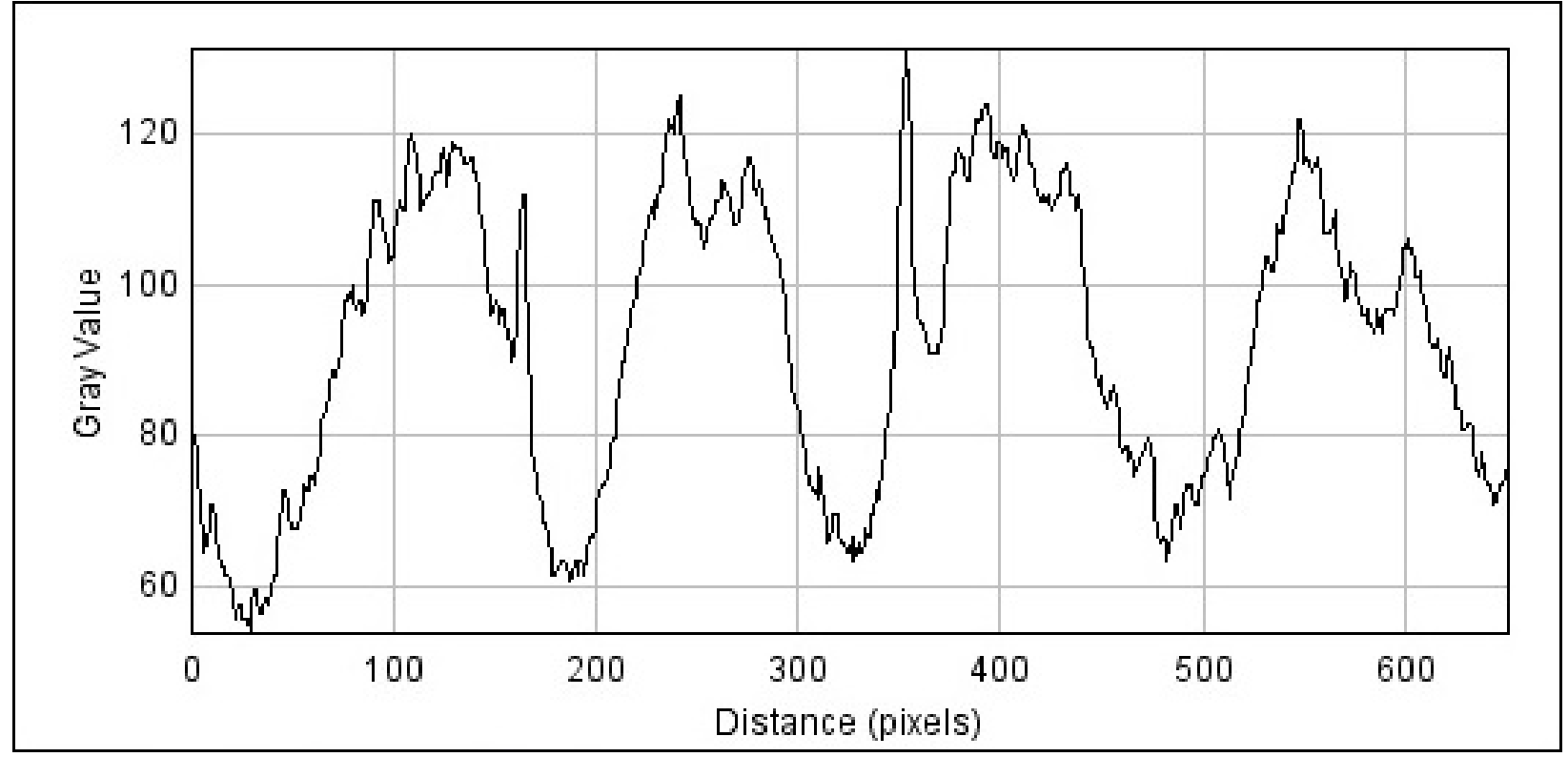


(g)

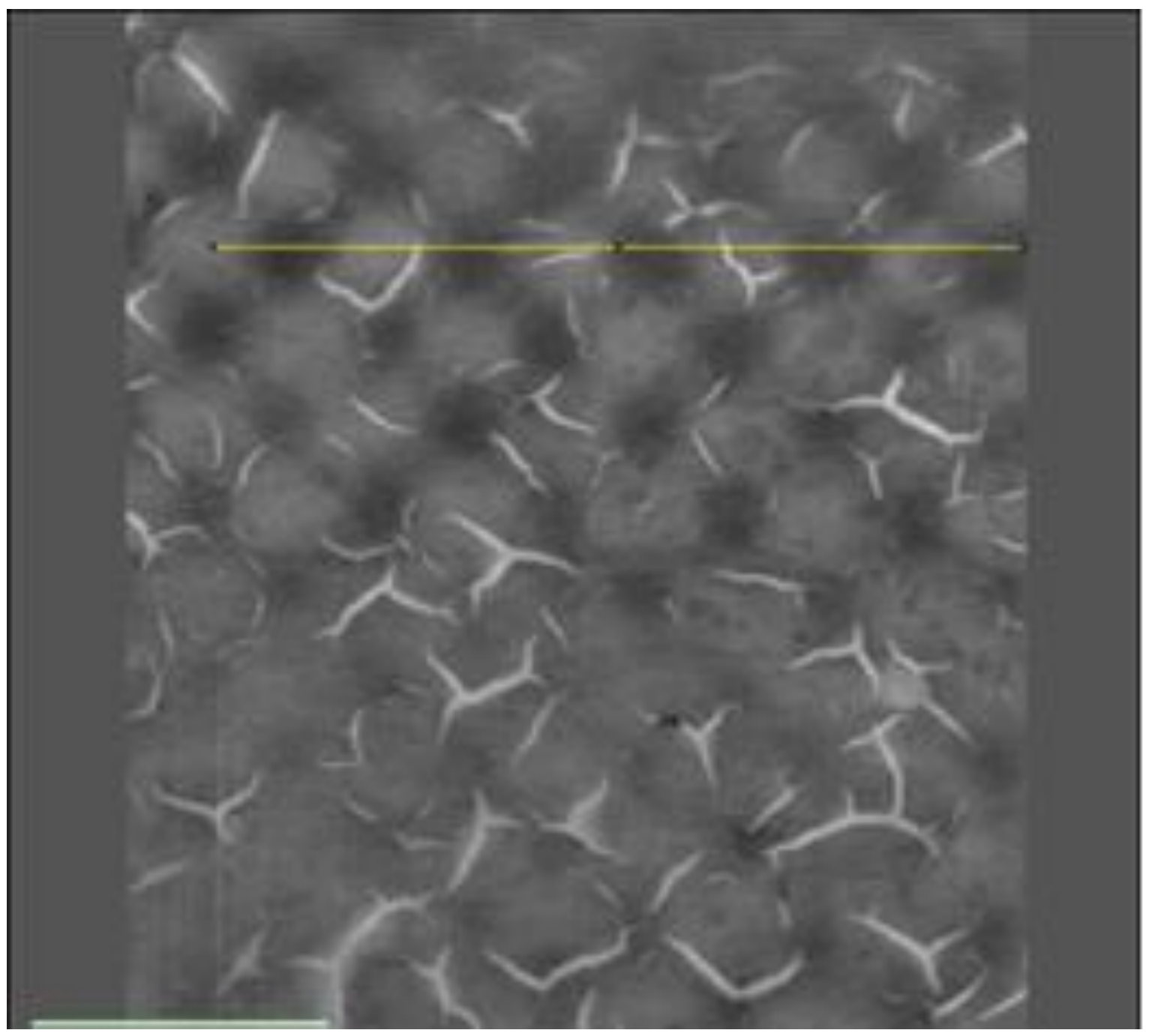

(h)

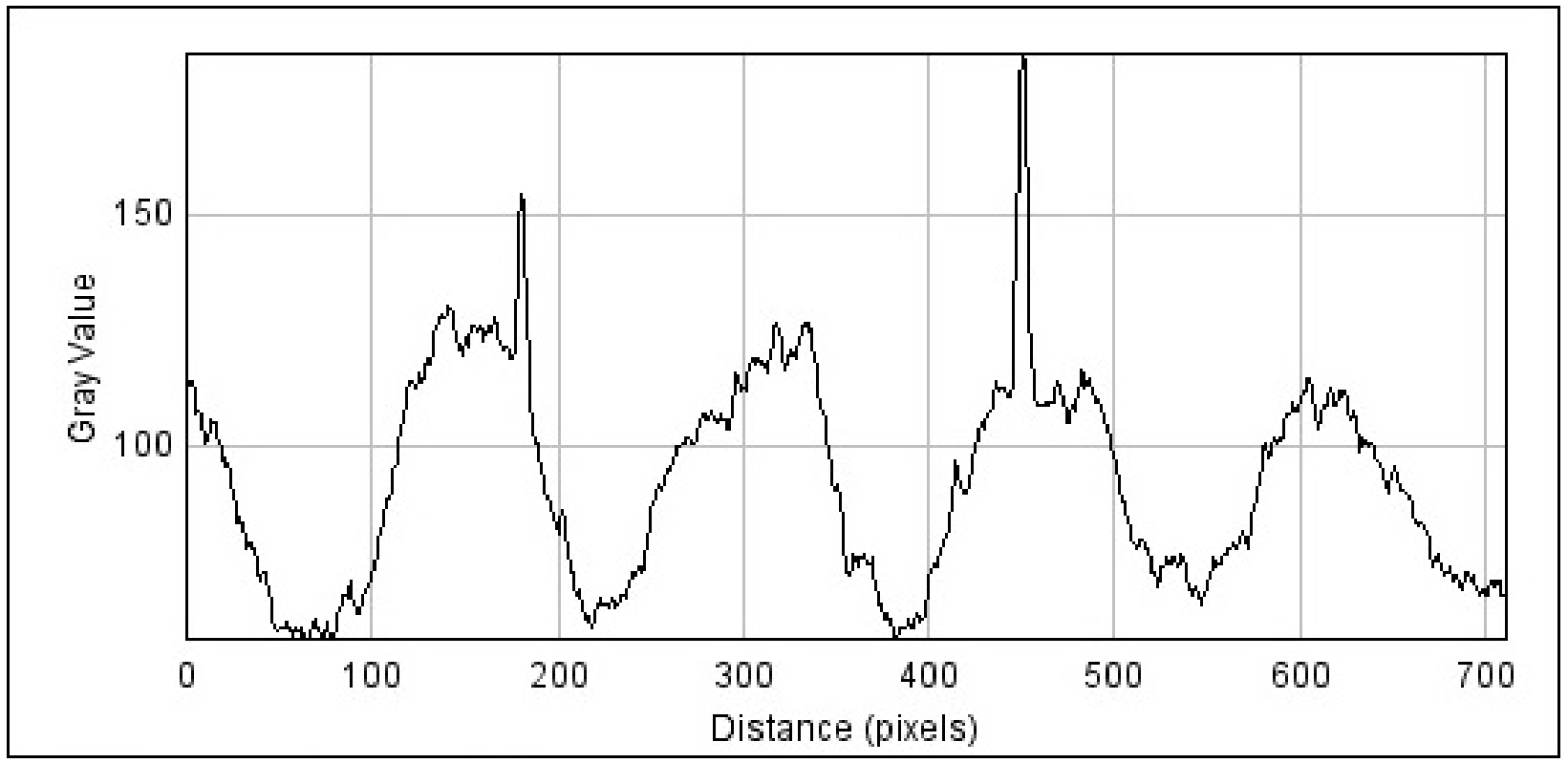




\section{DISCUSSION}

The various imaging techniques employed in this work have shown that the ink layer contains discrete platinum particles distributed through it, and, superficially, the internal structure across the ink layer thickness looks relatively uniform. However, the CXT images have revealed regular, periodic variations in X-ray absorption density in a 'polka-dot' like pattern akin to a hexagonally close-packed array. This pattern probably results from the screenprinting method used to produce the model layers of this study. In the screen-printing method the ink is squeezed out between the fibres of the screen itself and distributes across the backing. The screen becomes pressed down into the ink layer on the backing. After application of the ink the screen must be withdrawn, and the ink left in the screen must 'snapoff' from the ink on the substrate. The level of temporary adhesion of the screen behind the squeegee to the substrate depends upon the squeegee initial loading and speed, the tension in the screen mesh, and the gap size between the equilibrium (squeegee-free) position of the mesh and the substrate ${ }^{15}$. The tension in the mesh of the screen causes the mesh fibres to withdraw from the substrate. This leads to a suction-like force at the regions of the surface in contact with the fibres of the screen, and especially at the intersection points of the fibres. This suction may result in a reduced density in the ink at these sites. Increasing snap-off speed (e.g. due to an increased snap-off gap) is known to reduce the ink deposit ${ }^{15}$. The regular array of fibres in the screen and their intersections is thus probably responsible for the regular pattern in density distribution observed in the CXT images. The spacing of the holes in the mesh matches the spacing of the dots in the images obtained above.

The results reported above show that a very particular spatial pattern of entrapped mercury was observed in the CXT images, with mercury becoming preferentially entrapped in the high X-ray absorbing regions for the fresh sample. Due to the very high fractional entrapment of intruded mercury, as seen in Figure 5, the rise in X-ray absorbance density, due to the entrapment, is effectively a tracer for where the most mercury intrusion has occurred. For the fresh sample, the highest electron density element present initially is the platinum catalyst, since all other components are generally made up of elements in the earliest rows of the periodic table. The carbon support and platinum are always associated, so where there is enhanced electron density $(\mathrm{Pt})$ in the unfilled sample there will also be an enhanced amount 
of carbon. As the ink only consists of catalyst and ionomer, the only reason for enhanced platinum, and therefore catalyst, must be due to a partial depletion of the ionomer from this area. The ionomer in the ink would be expected to be mobile when the ink is printed and still wet. Hence, it seems likely that the white spot areas of enhanced electron density in the CXT images of fresh FC layer are due to a combination of relatively high catalyst concentration coupled with low ionomer concentration, whereas the dark areas are due to low catalyst concentration, but high ionomer concentration. When mercury is intruded into the catalyst layer, the areas that become more intensely X-ray absorbing are the areas not only with reduced amounts of ionomer, but also those with porosity that is still accessible. It is possible that the mercury is not entering the low X-ray absorbing areas because the enriched ionomer there is blocking access to many of the pores within that part of the layer. The low Pt density (dark) regions are enriched with ionomer and therefore may be expected to hold more water, if it can penetrate them.

\section{CONCLUSIONS}

The particular fabrication method for the cathode-side catalyst layer studied in this work has resulted in a regular patterning of the spatial arrangement of low and high density regions. A combination of mercury porosimetry and CXT has shown that the high density regions created when using a screen-printing process have relatively more accessible porosity. The variation in initial X-ray density in the fresh layers suggests this is due to segregation of the ionomer during the screen-printing process, which reduces the pore accessibility of the ionomer-enriched regions.

\section{ACKNOWLEDGEMENTS}

SPR and SM are grateful to the University of Nottingham for a PhD scholarship. 


\section{REFERENCES}

(1) Yoon, Y.-G.; Park, G.G. ; Yang, T.-H.; Han, J.H. ; Lee, W.Y.; Kim, C.S. Effect of pore structure of catalyst layer in a PEMFC on its performance. Int. J. Hydrogen Energy 2003, 28, 657.

(2) Ihm, J.W.; Ryu, H.; Bae, J.S.; Choo, W.K.; Choi, D.K. High performance of electrode with low Pt loading prepared by simplified direct screen printing process in PEM fuel cells. $J$. Mater. Sci. 2004, 39, 4647.

(3) Epting, W.K.; Gelb, J.; Litster, S. Resolving the three-dimensional microstructure of polymer electrolyte fuel cell electrodes using nanometer-scale X-ray computed tomography, Adv. Funct. Mater. 2012, 22, 555.

(4) Komini Babu, S.; Chung, H.; Wu, G.; Zelenay, P.; Litster, S. Modeling hierarchical nonprecious metal catalyst cathodes for PEFCs using multi-scale X-ray CT imaging. ECS Trans. 2014, 64, 281-292.

(5) Rigby, S.P.; Chigada, P.I.; Wang, J.; Wilkinson, S.K.; Bateman, H.; Al-Duri, B.; Wood, J.; Bakalis, S.; Miri, T. Improving the Interpretation of Mercury Porosimetry Data using Computerised X-ray Tomography and Mean-Field DFT, Chem. Engng Sci. 2011, 66, 2328.

(6) Ruffino, L.; Mann, R.; Oldman, R.; Stitt, E.H.; Boller, E.; Cloetens, P.; DiMichiel, M.; Merino, J. Using X-ray microtomography for characterisation of catalyst particle pore structure. Can. J. Chem. Engng 2005, 83, 132-139. 
(7) Scheiba, F.; Kunz, U.; Butsch, H.; Zils, S.; Fuess, H.; Roth, C. Imaging the electrodeGDL interface by a modified Wood's metal intrusion process. ECS Trans. 2010, 28, 85.

(8) Hellmuth, K.H.; Siitari-Kauppi, M.; Klobes, P.; Meyer, K.; Goebbels, J. Imaging and analyzing rock porosity by autoradiography and $\mathrm{Hg}$-porosimetry/X-ray computertomography-applications. Phys Chem Earth (A) 1999, 24, 569.

(9) Portsmouth, R.L.; Gladden, L.F. Mercury porosimetry as a probe of pore connectivity. Trans. Inst. Chem. Eng. 1992, 70A, 63.

(10) Tsakiroglou, C.D.; Payatakes, A.C. Effects of pore-size correlations on mercury porosimetry curves. J. Colloid Interface Sci. 1991, 146, 479.

(11) Rigby, S.P. A hierarchical structural model for the interpretation of mercury porosimetry and nitrogen sorption, J. Colloid Interface Sci. 2000, 224, 382.

(12) Wardlaw, N.C.; McKellar, M. Mercury porosimetry and the interpretation of pore geometry in sedimentary rocks and artificial models. Powder Technol. 1981, 29, 127.

(13) Kloubek, J. Hysteresis in porosimetry, Powder Technol. 1981, 29, 63.

(14) Ternan, M.; Mysak, L.P. Hysteresis caused by dimensional change of porous solids during mercury porosimetry. Powder Technol. 1987, 52, 29. 
(15) Fox, I.J.; Claypole, T.C.; Gethin, D.T. An experimental investigation into ink transfer using a roller squeegee in high-speed screen printing. Proc. Inst. Mech. Eng. Part E: J. Proc. Mech. Eng. 2003, 217, 307. 
GRAPHICAL ABSTRACT - TOC ONLY

CXT image slice of mercury (white) impregnated screen-printed fuel cell catalyst layer.

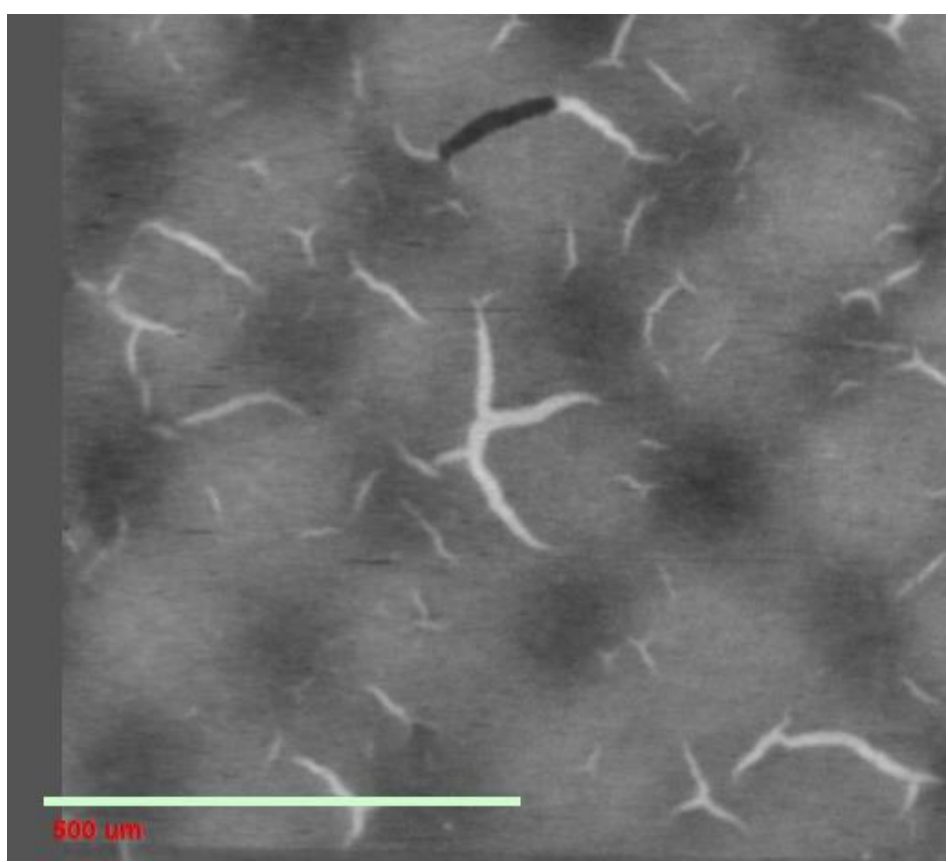

